\title{
Multiobjective Personnel Assignment Exploiting Workers' Sensitivity to Risk
}

\author{
Beatrice Lazzerini, Member, IEEE, and Francesco Pistolesi
}

\begin{abstract}
Every year 2.3 million people die worldwide due to occupational illnesses and accidents at work. By analyzing the workers' behavior when in the presence of risks managers could assign tasks to those workers who appear to be the most sensitive to risk being assigned and are thus more likely to exert more caution in the presence of that risk. This paper presents a novel multi-objective formulation of the personnel assignment problem, maximizing workers' sensitivity to risk, while minimizing cost and dislike for the task assigned. A worker's sensitivity to risk for a task is quantified by a new measure, carefulness, which stems from the worker's behavior and various human factors that affect the interaction with the risk. The problem is solved using a mixed evolutionary and multi-criteria decision making methodology. An approximation of the Pareto front is first generated through the non-dominated sorting genetic algorithm II. A hybrid decisional approach then exploits the technique for order of preference by similarity to ideal solution in order to select the Pareto-optimal solution that represents the nearest compromise to the decisionmaker's preferences. These preferences are derived through a fuzzy version of the analytic hierarchy process. The proposed framework was tested in four real-world scenarios related to manufacturing companies. The results show a significant increase in overall carefulness and a strong decrease in the dislike for the task assigned, with a modest increase in cost. The framework thus improves the work climate and reduces the risk occurrence and/or the impact on the workers' health.
\end{abstract}

Index Terms-Decision making, industrial safety, job assignment, multi-objective optimization, risk analysis.

\section{NOMENCLATURE}

$\begin{array}{ll}\mathcal{T} & \text { Set of tasks } \\ \mathcal{W} & \text { Set of workers } \\ \mathcal{R} & \text { Set of risks } \\ \mathcal{P} & \text { Set of preventive actions } \\ \mathcal{L} & \text { Set of levels of prevention } \\ \mathcal{F} & \text { Set of human factors } \\ i, j, k, q & \text { Index for tasks, workers, risks, preventive } \\ & \text { actions } \\ t_{i} & i \text {-th task } \\ w_{j} & j \text {-th worker } \\ r_{k} & k \text {-th risk } \\ h_{k} & \text { Hazardousness of risk } r_{k} \\ \eta_{i} & \text { Hazardousness of task } t_{i} \\ p_{q} & q \text {-th preventive action } \\ l_{q} & \text { Level of prevention of preventive action } p_{q} \\ \rho_{k j} & \text { Level of caution of worker } w_{j} \text { for risk } r_{k}\end{array}$

Manuscript received

The authors are with the Department of Information Engineering, University of Pisa, Largo L. Lazzarino, 1 - 56122 Pisa, Italy (e-mail: \{b.lazzerini, f.pistolesi\}@iet.unipi.it).

This research was supported by the PRA 2016 project "Analysis of Sensory Data: from Traditional Sensors to Social Sensors", funded by the University of Pisa.

$\begin{array}{ll}\tau_{i j} & \text { Level of caution of worker } w_{j} \text { for task } t_{i} \\ \mathbf{E} & \text { Exposure matrix } \\ \mathbf{B} & \text { Behaviour matrix } \\ \mathbf{S}^{\mathbf{j}} & \text { Global strategy matrix } \\ e_{i k} & \text { Generic element of the exposure matrix } \\ b_{k q} & \text { Generic element of the behavior matrix } \\ s_{k q}^{j} & \text { Generic element of the global strategy matrix } \\ & \text { of worker } w_{j} \\ \varphi_{j} & \text { Global task-independent human factors score } \\ & \text { of worker } w_{j} \\ \chi_{i j} & \text { Carefulness of worker } w_{j} \text { for task } t_{i} \\ a_{i j} & \text { Ability of worker } w_{j} \text { in performing task } t_{i} \\ z_{i j} & \text { Expertise human factor score of worker } w_{j} \\ & \text { for task } t_{i} \\ c_{i j} & \text { Cost of assigning worker } w_{j} \text { to task } t_{i} \\ d_{i j} & \text { Dislike of worker } w_{j} \text { for task } t_{i} \\ x_{i j} & \text { Decision variable equal to } 1 \text { if worker } w_{j} \text { is } \\ \mathbf{x} & \text { assigned to task } t_{i}, 0 \text { otherwise }\end{array}$

\section{INTRODUCTION}

$\mathbf{R}$ ISK is one of the major concerns when considering a personnel assignment problem, aimed at assigning the right people to the right tasks. This particularly holds in hazardous job assignments, where industrial safety is paramount. Unfortunately, this is extremely difficult because of the unquestionable subjectivity of the way people perceive, assess and control risks and hazardous situations [1].

There are two principal aspects influencing people's behavior in hazardous situations: risk perception and risk propensity [2]. Risk perception refers to a person's subjective way of estimating the characteristics and gravity of hazardous situations [3]-[7], while risk propensity refers to the person's tendency to take or avoid risks [8]-[10].

Risk perception is influenced by past experience and knowledge, past health status, psychological, social, political, and cultural factors, mood and emotions, personal knowledge about the risky conditions, trust in risk management institutions, age, and locus of control [11], [12], optimism bias [12], [13], etc. Likewise, risk propensity is influenced by personality and experience, cultural background, mood, feelings, education, job position, age, etc [14].

Although the factors affecting risk perception and risk propensity have been widely studied, people's behavior in dealing with risk has not been fully explained. Also, little is known about the interrelations between risk perception, risk propensity, and decisions involving risk [2].

Companies typically adopt specific risk programs [15] aimed at teaching workers to deal safely with risk. Risk aware- 
ness training is periodically repeated in order to continuously improve the training outcomes in terms of injury reduction, with consequent manifold capital investments by the employer. The training outcome can also be improved through a training method specifically tailored to the worker. In the literature, an association rule learning-based approach has thus been proposed in [14] to explore workers' risk sensitivity profiles. A risk sensitivity profile is an objective way to describe the accuracy of a worker in preventing one or more risks. In [16], an artificial neural network-based technique was proposed to classify workers into risk sensitivity profiles. Learning from constraints and evolutionary computation was also used in [17] to improve the classification accuracy. However, these solutions require significant economic resources.

Approximately $99 \%$ of companies in the EU are small and medium enterprises (SMEs) with a small number of employees. Altogether, SMEs employ 65 million people in the EU [18]. Outstanding manufacturing excellence and a small size are typical of Italian industry, which consists above all of family SMEs. With limited dimensions and financial resources, it is extremely important to keep costs as low as possible for these companies. Risk awareness training may involve considerable financial investments, which many SMEs are not prepared to make.

The best way to tackle these problems may be to consider workers' sensitivity to risk when deciding which worker is assigned to a task. This could make hazards less likely and less damaging.

Although workers' safety plays a key role in job assignment problems, companies typically consider objectives such as cost, work dislike (i.e., the worker's aversion for the task assigned), as well as, perhaps, other objectives. Personnel assignment should thus be set up as a multi-objective challenge that also takes workers' sensitivity to risk into account.

This paper presents a novel multi-objective formulation of the personnel assignment problem by exploiting workers' sensitivity to risk. The aim is to assign each task to the worker in such a way that he/she then has the safest way of interacting with risks.

A new measure, carefulness, is introduced to quantify a worker's sensitivity to risk towards a task. Its value is derived from the accuracy of the preventive actions a worker performs when exposed to the risks involved in the task, and from human factors related to the aspects influencing risk perception and risk propensity. The higher the worker's carefulness with respect to a task, the safer his/her behavior toward that task. Personnel assignment is therefore modeled as a multi-objective optimization (MOO) problem with three objectives: overall carefulness, overall cost and overall workers' dislike for the task assigned.

Two of the main disciplines dealing with MOO problems are multi-criteria decision-making (MCDM) and evolutionary multi-objective optimization (EMO). In this paper, a mixed EMO/MCDM resolution methodology is used to exploit the ability of EMO algorithms to widely explore the solution space, and the accuracy of MCDM techniques in selecting the solution representing the nearest compromise to the decisionmaker's preferences.
An EMO algorithm, i.e., the Non-dominated Sorting Genetic Algorithm II (NSGA-II) with purposely-defined constraint handling) is first used to generate Pareto-optimal solutions. Then, a hybrid MCDM technique selects the best solution in two steps: (i) a fuzzy version of the Analytic Hierarchy Process (F-AHP) is used to faithfully derive the decisionmaker's preferences for the objectives; (ii) the Technique for Order of Preference by Similarity to Ideal Solution (TOPSIS) considers each solution as an alternative of an MCDM problem and selects the best one using the preferences derived with FAHP.

The paper is organized as follows. Section II introduces multi-criteria decision making. Section III contains some preliminaries on multi-objective optimization, and Section IV describes the genetic algorithms fundamentals for both singleobjective and multi-objective optimization. Section V explains the concepts of sensitivity to risk and risk sensitivity profile. Section VI contains the formal model of the worker's carefulness. In Section VII, the problem formulation is given. Section VIII contains the details of the resolution methodology. In Section IX, four applications of the proposed optimization framework to real-world scenarios are discussed: one scenario involved personnel recruitment and the other three involved personnel reassignment. Finally, the conclusions are drawn in Section X.

\section{Multi-CRITERIA DECISION MAKING}

An MCDM problem is characterized by a goal, a set of criteria and a set of alternatives. Criteria and alternatives are called elements. Alternatives represent different choices available to the decision maker, while criteria are the different perspectives from which alternatives can be viewed. The goal consists in finding the best alternative with respect to all the criteria.

\section{A. Analytic Hierarchy Process}

AHP [19] is an MCDM method wherein elements are structured as a hierarchy. In general, criteria can be expressed in terms of sub-criteria. For the sake of clarity, here the simplest case is considered, where the hierarchy is composed of three levels: the uppermost containing the goal, the intermediate containing the criteria, and the lowest containing the alternatives. Each criterion is connected to the goal, and each alternative is connected to each individual criterion.

AHP ranks criteria with respect to each other and with reference to the goal. Alternatives are ranked with reference to each criterion. For each level of the hierarchy, AHP builds an $n \times n$ pairwise comparison matrix (hereafter $\mathrm{PCM}) \mathbf{P}=\left[p_{i j}\right]$ by comparing the elements with each other, with respect to each shared parent, i.e., each element of the upper level, where $n$ is the number of elements in the level, and $i, j \in\{1, \ldots, n\}$. The following conditions hold: $p_{i j}>0, p_{i j}=1 / p_{j i}$, and $p_{i i}=1 \forall i, j \in\{1, \ldots, n\}$. Coefficients $p_{i j}$, called preferences, estimate the preference of $i$ over $j$, and are typically expressed using the Saaty scale (see Table I).

Saaty proved in [20] that the relative weights of the compared elements are the components of the (normalized) 
principal eigenvector of a consistent PCM. An $n \times n$ PCM P is consistent if $p_{i j}=p_{i k} p_{k j} \forall i, j, k \in\{1, \ldots, n\}$. However, in real situations, this is not always achievable for PCMs based on the Saaty scale (Saaty matrices).

Hence, Saaty defined a consistency index, based on the principal eigenvalue $\lambda_{\max }$ of a Saaty matrix, defined as $C I \triangleq \frac{\lambda_{\max }-n}{n-1}$. The principal eigenvalue of a Saaty matrix always exists, and it holds $\lambda_{\max } \geq n$. A consistent matrix has $\lambda_{\max }=n$, so $C I \geq 0$, and the lower $C I$ the lower the inconsistency. To measure the level of inconsistency, AHP compares $C I$ with a random index $(R I)$ which represents the average consistency index of many $n \times n$ Saaty matrices randomly generated. The comparison is based on the consistency ratio $C R=\frac{C I}{R I}$. If $C R>0.1$ judgments have to be reformulated.

TABLE I

SAATY SCALE OF PREFERENCE

\begin{tabular}{|c|l|}
\hline Preference & Explanation \\
\hline 1 & Equally preferred \\
\hline 3 & Moderately preferred \\
\hline 5 & Strongly preferred \\
\hline 7 & Very Strongly preferred \\
\hline 9 & Extremely preferred \\
\hline $2,4,6,8$ & Intermediate values (compromises) \\
\hline
\end{tabular}

Once the relative weights have been computed, AHP determines the weight of each alternative with respect to the goal (i.e., its ranking) by multiplying the relative weight of the alternative with respect to each criterion by the relative weight of that criterion. These values (one for each criterion) are then summed and the highest-ranking alternative is chosen.

AHP has been criticized because it entails rank reversal, i.e., possible changes within the ranking whenever an alternative is removed or a new alternative is added [21]. However, in this paper, AHP is used just to prioritize the criteria (i.e., the objectives).

\section{B. Fuzzy AHP}

Human judgements are usually affected by imprecision and vagueness. Fuzzy set theory [22] can effectively deal with this problem. A fuzzy set is defined as $F=\left\{\left(x, \mu_{F}(x)\right), x \in \mathbb{U}\right\}$, where $\mathbb{U}$ is the universe of discourse and $\mu_{F}$ is the membership function such that $x \mapsto \mu_{F}(x)$, where $\mu_{F}(x) \in[0,1]$. The set $\left\{x: \mu_{F}(x)>0\right\}$ is the support of fuzzy set $F$.

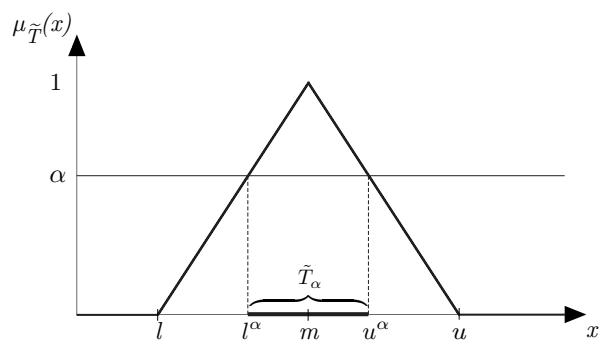

Fig. 1. Membership function of a TFN $\tilde{T}$, and its alpha-cut $\tilde{T}_{\alpha}$.

A fuzzy number is a convex and normalized fuzzy set defined on $\mathbb{R}$. Triangular fuzzy numbers (TFNs) are widely used to express human judgments. Formally, given $l, m, u \in \mathbb{R}$, such that $l \leq m \leq u$, the membership function $\mu_{\tilde{T}}(x)$ of a
TFN $\tilde{T}$, shown in Fig. 1, assumes values such that: $\mu_{\tilde{T}}(x)=0$ if $x<l \vee x>u, \mu_{\tilde{T}}(x)=\frac{x-l}{m-l}$ if $l \leq x \leq m, \mu_{\tilde{T}}(x)=\frac{u-x}{u-m}$ if $m \leq x \leq u$.

An alternative representation of $\tilde{T}$, based on the interval of confidence (or alpha-cut), is $\tilde{T}_{\alpha}=\left[l^{\alpha}, u^{\alpha}\right]=[(m-l) \alpha+$ $l,-(u-m) \alpha+u], \forall \alpha \in[0,1]$ (see Fig. 1).

F-AHP deals with uncertainty and vagueness by substituting Saaty's scale with a fuzzy version based on TFNs from $\tilde{1}$ to $\tilde{9}$. More rigorously, let $\tilde{\mathbf{P}}$ be an $n \times n$ PCM containing TFNs $\tilde{p}_{i j}^{\alpha}=\left[p_{i j_{l}}^{\alpha}, p_{i j_{u}}^{\alpha}\right]$, and let $\tilde{\mathbf{x}}$ be a non-zero $n \times 1$ vector containing fuzzy numbers $\tilde{x}_{i}=\left[x_{i_{l}}^{\alpha}, x_{i_{u}}^{\alpha}\right]$. A fuzzy eigenvalue $\tilde{\lambda}$ is a fuzzy number that is a solution of $\tilde{\mathbf{P}} \tilde{\mathbf{x}}=\tilde{\lambda} \tilde{\mathbf{x}}$. In order to compute the principal eigenvector, matrix $\tilde{\mathbf{P}}$ needs to be defuzzified. Defuzzification maps a fuzzy set into a number. There are several ways to perform defuzzification. For instance, $\tilde{\mathbf{P}}$ can be defuzzified by introducing a coefficient $\zeta \in[0,1]$, called index of optimism, used to perform a convex combination for each element of $\tilde{\mathbf{P}}$, thus obtaining $\hat{\mathbf{P}}=\left[\hat{p}_{i j}^{\alpha}\right]=\left[\zeta p_{i j_{u}}^{\alpha}+(1-\zeta) p_{i j_{l}}^{\alpha}\right]$. The principal eigenvector of $\hat{\mathbf{P}}$ is then calculated as in classic AHP.

\section{Technique for Order of Preference by Similarity to Ideal Solution (TOPSIS)}

TOPSIS is an MCDM technique [23], [24]. Consider a problem with $n$ alternatives and $m$ criteria. TOPSIS needs an $n \times m$ decision matrix

$$
\mathbf{H}=\begin{gathered}
C_{1} \\
A_{1} \\
A_{2} \\
\vdots \\
A_{n}
\end{gathered}\left(\begin{array}{cccc}
h_{11} & h_{12} & \cdots & h_{1 m} \\
h_{21} & h_{22} & \cdots & h_{2 m} \\
\vdots & \vdots & \ddots & \vdots \\
h_{n 1} & h_{n 2} & \cdots & h_{n m}
\end{array}\right),
$$

where $A_{i}$ is the $i$-th alternative, $C_{j}$ is the $j$-th criterion, and $h_{i j}$ is the performance of $A_{i}$ with respect to $C_{j}$, where $i \in\{1, \ldots, n\}$ and $j \in\{1, \ldots, m\}$. TOPSIS also considers a vector $\boldsymbol{\omega}=\left(\omega_{1}, \ldots, \omega_{m}\right)$, where $\sum_{j=1}^{m} \omega_{j}=1$, which contains the weights of the criteria. The weights of the criteria and the performance of each alternative with respect to each criterion are either directly expressed by experts [25], or they result from an automatic prioritization process [26]. To select the best alternative, TOPSIS performs the following steps:

1) construct the normalized decision matrix $\mathbf{R}=\left[r_{i j}\right]$, where $r_{i j}=h_{i j} / \sqrt{\sum_{i=1}^{n} h_{i j}^{2}}$;

2) build the weighted normalized decision matrix $\mathbf{V}$ whose generic element is $v_{i j}=\omega_{j} r_{i j}$;

3) determine the ideal solutions, i.e., utopia and nadir. Let $\Omega_{B}$ and $\Omega_{C}$ contain the indexes of benefit and cost criteria, respectively. Utopia and nadir are defined, respectively, as $A^{+}=\left(a_{1}^{+}, \ldots, a_{m}^{+}\right)$and $A^{-}=\left(a_{1}^{-}, \ldots, a_{m}^{-}\right)$, where $a_{j}^{+}=\max _{i=1, \ldots, n} v_{i j}$ for $j \in \Omega_{B}$ or $a_{j}^{+}=$ $\min _{i=1, \ldots, n} v_{i j}$ for $j \in \Omega_{C}$, and $a_{j}^{-}=\min _{i=1, \ldots, n} v_{i j}$ for $j \in \Omega_{B}$ or $a_{j}^{-}=\max _{i=1, \ldots, n} v_{i j}$ for $j \in \Omega_{C}$;

4) calculate the Euclidean distance of each alternative from utopia and nadir, respectively, as $D_{i}^{+}=$ $\sqrt{\sum_{j=1}^{m}\left(v_{i j}-a_{j}^{+}\right)^{2}}$ and $D_{i}^{-}=\sqrt{\sum_{j=1}^{m}\left(v_{i j}-a_{j}^{-}\right)^{2}}$; 
5) calculate the relative closeness coefficient of each alternative to utopia as $R C L_{i}^{+}=D_{i}^{-} /\left(D_{i}^{+}+D_{i}^{-}\right)$, where the greater $R C L_{i}^{+}$the better $A_{i}$;

6) rank alternatives in descending order of relative closeness coefficients.

The alternative $A_{k}$ such that $k=\arg \max _{i} R C L_{i}^{+}$is the best.

\section{Multi-OBjective OPtimization}

\section{A. Fundamentals}

MOO aims to simultaneously optimize multiple objectives. Formally, an MOO problem can be written as

$$
\underset{\mathbf{x}}{\operatorname{Minimize}} \mathbf{f}(\mathbf{x})=\left[f_{1}(\mathbf{x}), f_{2}(\mathbf{x}), \ldots, f_{k}(\mathbf{x})\right]
$$

subject to:

$$
\begin{aligned}
& g_{i}(\mathbf{x}) \leq 0, \quad i=1, \ldots, m \\
& h_{j}(\mathbf{x})=0, \quad j=1, \ldots, n
\end{aligned}
$$

where $\mathbf{x} \in \mathbb{R}^{p}, k \geq 2$ is the number of objectives, $m$ and $n$ are, respectively, the number of inequality and equality constraints that define the feasible region $\mathcal{X} \subset \mathbb{R}^{p}$. The vector-valued objective function $\mathbf{f}: \mathbb{R}^{p} \rightarrow \mathbb{R}^{k}$, defined as $\mathbf{f}(\mathbf{x})=\left[f_{1}(\mathbf{x}), \ldots, f_{k}(\mathbf{x})\right]$, contains the functions to minimize as components. Each $\mathrm{x} \in \mathcal{X}$ is a feasible solution.

\section{B. Pareto dominance and Pareto optimality}

In general, MOO problems have no feasible solution for minimizing all the objective functions at the same time. The concepts of Pareto dominance and Pareto-optimal solution are therefore introduced. Given the minimization problem (2)-(4), consider two feasible solutions $\mathrm{x}^{1}, \mathrm{x}^{2} \in \mathcal{X}$. Solution $\mathrm{x}^{1}$ is said to dominate $\mathbf{x}^{\mathbf{2}}$, in symbols $\mathbf{x}^{\mathbf{1}} \prec \mathbf{x}^{2}$, if $f_{i}\left(\mathbf{x}^{\mathbf{1}}\right) \leq$ $f_{i}\left(\mathbf{x}^{2}\right) \forall i \in\{1, \ldots, k\}$, and $f_{j}\left(\mathbf{x}^{\mathbf{1}}\right)<f_{j}\left(\mathbf{x}^{\mathbf{2}}\right)$ for at least one index $j \in\{1, \ldots, k\}$. A Pareto-optimal solution is a feasible solution that cannot be improved with respect to any objective without degrading at least one of the other objectives. The set of images of Pareto-optimal solutions in the objective space is called the Pareto front.

\section{GenetiC ALGORITHMS}

\section{A. Overview}

A genetic algorithm (GA) is a heuristic algorithm based on the principles of natural selection and biological evolution [27]. GAs are usually exploited to solve complex optimization problems that are hard or impractical to deal with analytically. GAs represent a solution to the problem through encoding (e.g., bit/integer string, or real-valued vector). A solution is called individual and consists of a chromosome, whose components are called genes.

The first step of a GA consists in generating a set of candidate solutions to the problem, i.e., a set of individuals, forming a population. The goodness of each individual is evaluated by a fitness function: for a minimization problem, the lower the fitness, the better the individual. A selection phase enables individuals with the best fitness be selected for reproduction; the selected individuals form the mating pool.
Then, typically, pairs of individuals (parents) from the mating pool are recombined through the crossover operator. The mutation operator can also be applied. Individuals generated by reproduction (offspring) replace (part of) the previous population.

The process is iterated until a terminating condition is met, e.g., a maximum number of generations. The individual with the best fitness in the last population, or throughout all populations, is typically chosen as the optimal solution.

\section{B. Genetic operators used in the resolution methodology}

1) Partially matched crossover: Partially Matched Crossover (PMX) preserves offspring feasibility within a constrained problem [28]. PMX is illustrated in Fig. 2, where chromosomes are represented as integer strings and feasibility means no duplicated gene. From an operational point of view,

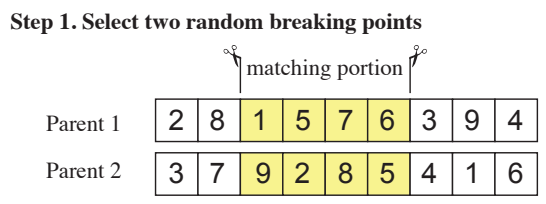

Step 2. Exchange the genes position-wise in the matching portion

\begin{tabular}{ll|l|l|l|l|l|l|l|l|} 
Offspring 1 & $(2$ & 8 & 9 & 2 & 8 & 5 & 3 & 9 & 4 \\
Offspring 2 & 3 & 7 & 1 & 5 & 7 & 6 & 4 & 1 & $(6$ \\
\hline
\end{tabular}

Step 3. Determine the mapping relationship

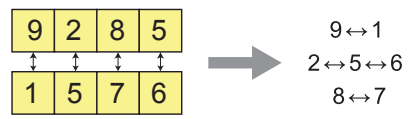

Step 4. Repair offspring using the mapping relationship

\begin{tabular}{l|l|l|l|l|l|l|l|l|l|} 
Offspring 1 & 6 & 7 & 9 & 2 & 8 & 5 & 3 & 1 & 4 \\
\hline
\end{tabular}

\begin{tabular}{l|l|l|l|l|l|l|l|l|l|} 
Offspring 2 & 3 & 8 & 1 & 5 & 7 & 6 & 4 & 9 & 6 \\
\hline
\end{tabular}

Fig. 2. Partially Matched Crossover.

consider two parents. Two breaking points are generated randomly along the chromosomes (step 1 in Fig. 2). Genes between the breaking points form the matching portion. Position-wise gene exchanges are then performed within the matching portion to generate two offspring (step 2 in Fig. 2). The mapping of the exchanged genes is called the mapping relationship (step 3 in Fig. 2). Consider each offspring. Each gene outside the matching portion that generates a duplicate (circled in step 2 in Fig. 2) is repaired by replacing it with the corresponding value in the mapping relationship. Repairing is shown in step 4 in Fig. 2.

2) Swap mutation: Swap mutation is the randomized gene exchange within chromosomes. For each chromosome, two genes are chosen at random and are exchanged.

\section{The NSGA-II algorithm}

Genetic algorithms can be used to solve multi-objective problems. One of the most efficient is NSGA-II [29].

NSGA-II starts by randomly generating an initial population $P_{0}$ of $n$ individuals, and associates each individual with a non-domination rank: rank 1 for the best level, rank 2 for the 
next best level, and so on. NSGA-II first determines the nondominated individuals in $P_{0}$ and assigns them all rank 1; these individuals belong to the first front. Then, individuals from the first front are neglected in order to find individuals with rank 2 , i.e., those in the next front, and so on.

At iteration $t$, NSGA-II generates an offspring population $Q_{t}$ of $n$ individuals, by selecting from the parent population $P_{t}$ using binary tournament selection. In a binary tournament two members of $P_{t}$ are selected randomly and the strongest of the two is added to the mating pool. Then, crossover and mutation operators are applied. Parent and offspring populations are merged into a new population $P_{e x t}=P_{t} \cup Q_{t}$. Each of the $2 n$ individuals in $P_{\text {ext }}$ is associated with its non-domination rank and, accordingly, $P_{e x t}$ is divided into fronts. Within each front, the crowding distance is used to estimate the density of individuals in each individual's neighborhood. Crowding distance is the sum of the distances from an individual to its closest individual along each objective. NSGA-II sorts individuals within a front according to their crowding distances. The new parent population $P_{t+1}$ is obtained from $P_{\text {ext }}$ by discarding the worst $n$ individuals, firstly considering sorting among fronts, and then among individuals within the same front. NSGA-II iterates for a specified number of generations.

\section{WORKER'S SENSITIVITY TO RISK AND RISK SENSITIVITY PROFILE}

\section{A. Risk perception, risk propensity and human factors}

Let $\mathcal{F}$ be a set of human factors (or simply factors) whose correlation with risk perception and risk propensity has been reported in the sociology and psychology literature [11]-[13]. Human factors are here divided into personal, psychological and task-related. Personal factors refer to factors related to an individual's past history and work experience. Personal and psychological factors are uniquely correlated with the worker, irrespectively of the task performed. Differently, taskrelated factors assume values that are dependent on the task performed. Human factors are summarized in Table II.

TABLE II

HUMAN FACTORS CORRELATED WITH RISK PERCEPTION AND RISK PROPENSITY

\begin{tabular}{|c|l|}
\hline Personal & $\begin{array}{l}\text { age, level of education, income, number of past } \\
\text { industrial injuries and diseases, overall severity } \\
\text { of the suffered industrial injuries and diseases, } \\
\text { injury frequency, personal knowledge of the } \\
\text { risks of the workplace, delay of occurrence of } \\
\text { the risk effects, perceived level of work control, } \\
\text { perceived level of risk control }\end{array}$ \\
\hline Task-related & acquired expertise, dislike for task \\
\hline Psychological & anxiety level, self-esteem level, worry level \\
\hline
\end{tabular}

Consider a worker $w$. Each human factor $f \in \mathcal{F}$ is quantified by a human factor value $\phi_{f, w}$ (hereafter, simply referred to as value) belonging to a domain $\mathcal{D}_{f}$. The set $\bigcup_{f \in \mathcal{F}}\left(f, \phi_{f, w}\right)$, containing (human factor, value) pairs, one for each human factor, is the human factors configuration of $w$.

\section{B. From behavioral strategies to risk sensitivity profiles}

Let us consider a simple work environment characterized by a unique risk $r$. Let set $\mathcal{P}_{r}$ contain all the preventive actions a worker can perform to prevent $r$. A preventive action mitigates a risk in the sense that it can reduce the probability of the risk materializing and/or lower the risk impact, i.e., the severity of the impact on the worker's health. The higher the level of reduction, the more effective the action is in preventing the risk. In general, it is not possible to reduce the probability of a risk materializing to zero, nor can its impact be reduced to zero.

Different workers exposed to the same risk generally choose different preventive actions to protect themselves. With reference to risk $r$ and worker $w$, the subset of preventive actions chosen by $w$ is called strategy toward $r$, and represents how effective the worker's behavior is in preventing $r$. Also, the higher the values assumed by the human factors that are directly correlated with higher values of risk perception (see Section VI-C), the more conscious the worker is in dealing with risk. This essentially means that a risk sensitivity profile composed of highly-effective preventive actions and high values for the human factors directly correlated with higher values of risk perception, denotes a careful worker due to his/her high sensitivity to risk. The model proposed in this paper assumes that if a worker has a high sensitivity to risk, he/she will be more careful, which makes the risk less likely to occur and/or will lead to a less severe injury.

The risk sensitivity profile $\Pi_{r, w}$ of worker $w$ for risk $r$ consists of two elements: the worker's strategy toward the risk and the worker's human factors configuration, i.e.,

$$
\Pi_{r, w}=\left\{\mathcal{P}_{r, w}, \bigcup_{f \in \mathcal{F}}\left(f, \phi_{f, w}\right)\right\},
$$

where $\mathcal{P}_{r, w} \subseteq \mathcal{P}_{r}$ is the set of preventive actions worker $w$ performs when exposed to risk $r$.

The risk sensitivity profile of a worker is fundamental in modeling the worker's level of caution with respect to each risk he/she is exposed to, as the level of caution stems from the worker's strategy in dealing with the considered risk. In turn, the levels of caution of a worker (one for each risk) and the human factors configuration are used to derive the worker's carefulness with respect to a task, i.e., a new measure, which is formalized in the next section and which expresses the worker's suitability level (in terms of safety) to the risks to which he/she is exposed while performing the task.

\section{MODELING WORKER'S CAREFULNESS}

\section{A. Basic elements of the model}

In this section, the concept of carefulness is rigorously formalized. To make the model easier to understand, its basic elements are first discussed from an intuitive point of view.

Within a workplace, each task is characterized by a set of risks. Each risk has a level of hazardousness; the higher the hazardousness, the more likely and/or dangerous the risk. A worker performing a task can reduce the probability of each risk materializing, as well as the risk impact, thanks to a behavioral strategy consisting of one or more preventive actions. Each preventive action has a level of prevention. 
The higher the level of prevention, the safer the worker's interaction with a risk. Thanks to the strategy, a worker's level of caution with respect to a risk is derived by analyzing the number of preventive actions he/she performs for each level of prevention. Such numbers are then aggregated through a weighted sum, wherein each level of prevention has a weight.

A worker's carefulness for a task measures the level of safety resulting from assigning the worker to the task. In the proposed model, this extent of safety stems from:

- the worker's level of caution with respect to each risk of the task;

- the worker's human factors configuration.

\section{B. Worker's level of caution with respect to risks and tasks}

Let us consider a set of tasks $\mathcal{T}=\left\{t_{1}, \ldots, t_{|\mathcal{T}|}\right\}$ and a set of risks $\mathcal{R}=\left\{r_{1}, \ldots, r_{|\mathcal{R}|}\right\}$, where the symbol $|\cdot|$ denotes the cardinality of the set. Let $\mathbf{E}$ be a $|\mathcal{T}| \times|\mathcal{R}|$ matrix, called exposure matrix, whose generic element $e_{i k}=1$ when performing task $t_{i}$ exposes to risk $r_{k}$, otherwise $e_{i k}=0$, where, from now on, $i \in\{1, \ldots,|\mathcal{T}|\}$ and $k \in\{1, \ldots,|\mathcal{R}|\}$.

Each risk $r_{k} \in \mathcal{R}$ has a risk hazardousness $h_{k} \in(0,1]$, which accounts for the probability of the risk materializing and its impact. The higher the risk hazardousness the more likely a risk and/or the more serious its effects on the workers' health. Generally, the risk hazardousness is defined by an expert in risk assessment.

Each task $t_{i} \in \mathcal{T}$ is associated with a task hazardousness $\eta_{i} \in(0,1]$, here defined as:

$$
\eta_{i}=\max _{\substack{k \in\{1, \ldots,|\mathcal{R}|\} \\ e_{i k}=1}} h_{k}
$$

Using the max operator in (6), the task hazardousness $\eta_{i}$ is equal to the risk hazardousness $h_{k}$ of the most dangerous risk workers deal with while performing task $t_{i}$. In fact, whatever the task, the greatest danger for workers broadly comes from the impact the most serious risk may have on their health.

As stated in Section V-B, preventive actions make a risk less likely to occur and/or they can reduce its impact. Rigorously, if $\mathcal{P}=\left\{p_{1}, \ldots, p_{|\mathcal{P}|}\right\}$ is the set of all the preventive actions for all the risks, let $\mathcal{P}_{r_{k}}$ be the set of actions that can prevent risk $r_{k}$, where $\mathcal{P}_{r_{k}} \subset \mathcal{P}$. Let $\mathbf{B}$ be an $|\mathcal{R}| \times|\mathcal{P}|$ matrix, called behavior matrix, whose generic element is:

$$
b_{k q}= \begin{cases}1 & \text { if } r_{k} \text { can be prevented by performing } p_{q} \\ 0 & \text { otherwise. }\end{cases}
$$

Depending on the probability/impact reduction capability, each preventive action $p_{q}$, where $q \in\{1, \ldots,|\mathcal{P}|\}$, is associated with a level of prevention $l_{q} \in \mathcal{L}=\left\{1, \ldots, \ell_{\max }\right\}$. High levels of prevention denote preventive actions that lead to a considerable reduction in the probability of occurrence and/or impact of the risks $r_{k}$, such that $b_{k q}=1$. Note that $\ell_{\max }$ stands for the maximum level of prevention by which preventive actions can be characterized. In general, even possible preventive actions $p_{q}$ with $l_{q}=\ell_{\max }$, cannot fully prevent the risks $r_{k}$ for which $b_{k q}=1$. Preventive actions are associated with levels of prevention by experts in risk assessment, who evaluate the ability of each preventive action to make the related risks less likely as well as reducing their impact.
As explained in Section $\mathrm{V}-\mathrm{B}$, a worker $w_{j} \in \mathcal{W}$ performs a subset of the actions in $\mathcal{P}_{r_{k}}$ when exposed to risk $r_{k} \in \mathcal{R}$ : this subset of actions is the strategy of $w_{j}$ toward $r_{k}$. The strategies of worker $w_{j}$ with respect to each risk $r_{k}$ make up the global strategy of $w_{j}$. Formally, the global strategy of $w_{j}$ is represented by an $|\mathcal{R}| \times|\mathcal{P}|$ matrix $\mathbf{S}^{\mathbf{j}}$, called global strategy matrix, whose rows and columns are related to risks and preventive actions, respectively. The generic element $s_{k q}^{j}$ of $\mathbf{S}^{\mathbf{j}}$ is:

$$
s_{k q}^{j}= \begin{cases}1 & \text { if } w_{j} \text { performs } p_{q} \text { when exposed to } r_{k} \\ 0 & \text { otherwise. }\end{cases}
$$

A global strategy matrix must contain only valid strategies, hence the strategy worker $w_{j}$ uses to prevent risk $r_{k}$ (represented by the $k$-th row $\mathbf{s}_{\mathbf{k}}^{\mathbf{j}}$ of $\mathbf{S}^{\mathbf{j}}$ ) can be exclusively made up of preventive actions in $\mathcal{P}_{r_{k}}$, i.e., more rigorously,

$$
b_{k q}-s_{k q}^{j} \neq-1, \quad \forall q=1, \ldots,|\mathcal{P}|,
$$

where $b_{k q}$ is an element of the behavior matrix, whose value is defined in (7).

For the sake of clarity, consider house painting as a sample task that exposes a worker to various risks. For example, consider an accidental fall or shoulder pain due to poor posture. A different risk hazardousness is associated with each risk, based on the seriousness of its effects on the worker's health in the specific working context. Accordingly, the task hazardousness is equal to the higher risk hazardousness of the two risks involved. Let us suppose that possible preventive actions for the fall risk are, e.g., use of safety snap hooks, checking the stability of the platform/ladder, refraining from climbing up ladders, shelves, stacks of boxes, etc., refraining from rapid movements, use of safety shoes, etc. Likewise, suppose preventive actions for shoulder pain due to poor posture are to keep the shoulders in alignment, to avoid maintaining the same position for a long time, to keep the upper back and neck straight, etc. Each action has a specific level of prevention depending on its capacity to prevent the corresponding risk. Suppose we have three levels of prevention, labeled 1, 2 and 3 , corresponding to low, medium and high, respectively. With reference to the fall risk, the highest level of prevention may be associated, for example, with fastening the safety snap hook because of its ability to reduce the risk probability and impact: even if a worker falls, the snap hook prevents him/her from hitting the ground. On the other hand, an expert may associate checking the stability of the platform/ladder with a lower level of prevention (e.g., medium) because this action actually makes the risk less likely to happen but has no effect on the risk impact. A worker's strategy toward a risk is a subset of all the preventive actions of that risk. A worker's global strategy is the union of the strategies for the two risks.

Now, more formally, consider a worker $w_{j}$ and his/her global strategy in the global strategy matrix $\mathbf{S}^{\mathbf{j}}$. We define the vector-valued function $\#:\{0,1\}^{|\mathcal{R}| \times|\mathcal{P}|} \rightarrow \mathbb{Z}_{+}^{|\mathcal{R}| \times|\mathcal{L}|}$, which takes an $|\mathcal{R}| \times|\mathcal{P}|$ global strategy matrix $\mathbf{S}^{\mathbf{j}}$ and obtains, for each strategy (i.e., for each risk), the number of preventive actions per level of prevention. The image of the function \#, 


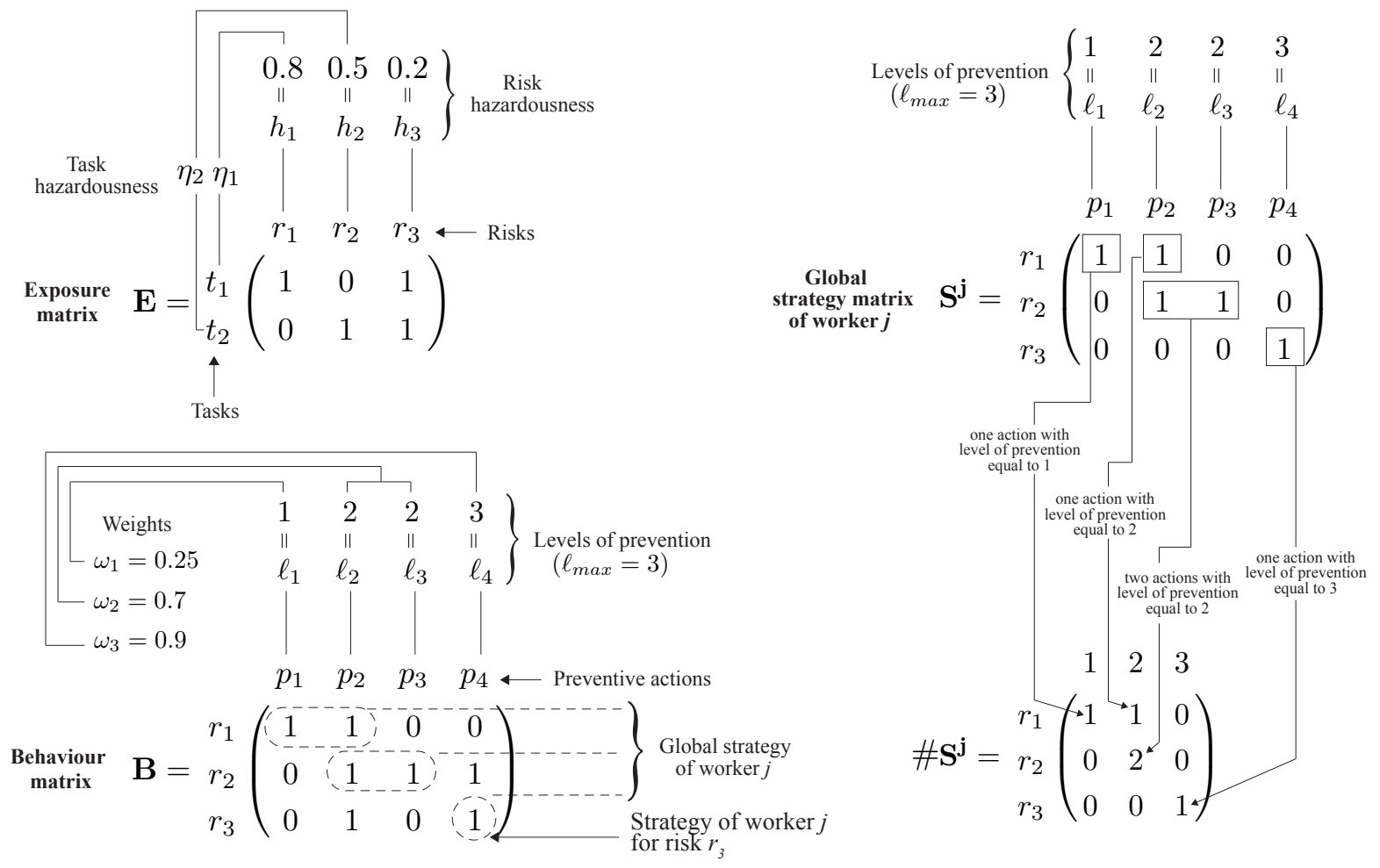

Fig. 3. Elements of the carefulness modeling for a scenario characterized by two tasks $\left(t_{1}\right.$ and $\left.t_{2}\right)$, three risks $\left(r_{1}, r_{2}\right.$ and $\left.r_{3}\right)$ and four preventive actions $\left(p_{1}, p_{2}, p_{3}\right.$ and $\left.p_{4}\right)$. The upper left corner contains the exposure matrix $\mathbf{E}$, and the hazardousness of each risk $\left(h_{1}, h_{2}\right.$ and $\left.h_{3}\right)$ and each task $\left(\eta_{1}\right.$ and $\left.\eta_{2}\right)$. The bottom left corner contains the behavior matrix $\mathbf{B}$, the level of prevention of each preventive action $\left(\ell_{1}, \ell_{2}, \ell_{3}\right.$ and $\left.\ell_{4}\right)$ and the corresponding weights. The upper right corner contains the global strategy matrix $\mathbf{S}^{\mathbf{j}}$ of worker $w_{j}$, derived from the elements enclosed by a dashed line in the behavior matrix $\mathbf{B}$. In matrix $\mathbf{S}^{\mathbf{j}}$, a solid line frame encloses preventive actions with the same level of prevention. The bottom right corner shows the image $\# \mathbf{S}^{\mathbf{j}}$ of function $\#$ applied to matrix $\mathbf{S}^{\mathbf{j}}$. Each column is associated with a level of prevention (i.e., 1, 2 and 3).

denoted by $\# \mathbf{S}^{\mathbf{j}}$, is an $|\mathcal{R}| \times|\mathcal{L}|$ matrix whose generic element $\left[\# \mathbf{S}^{\mathbf{j}}\right]_{k \ell}$ is defined as:

$$
\left[\# \mathbf{S}^{\mathbf{j}}\right]_{k \ell}=\sum_{\substack{q=1, \ldots,|\mathcal{P}| \\ l_{q}=\ell}} s_{k q}^{j} .
$$

In (10), the count of $\ell$-level actions performed by $w_{j}$ when exposed to $r_{k}$ is assigned to element $\left[\# \mathbf{S}^{\mathbf{j}}\right]_{k \ell}$ of matrix $\# \mathbf{S}^{\mathbf{j}}$. A weight $\omega_{\ell} \in(0,1]$ is then associated with each level of prevention $\ell \in \mathcal{L}$, typically by an expert in risk assessment: the higher the weight of a preventive action, the better it mitigates a risk. To help the reader, the modeling elements formalized so far are graphically summarized in Fig. 3 for a simple scenario.

The level of caution $\rho_{k j}$ of worker $w_{j}$ with respect to risk $r_{k}$ is here defined as a weighted sum of the count of preventive actions used by the worker for each level of prevention, normalized so that $\rho_{k j} \in[0,1]$, hence:

$$
\rho_{k j}=\frac{\sum_{\ell=1}^{\ell_{\max }} \omega_{\ell} \cdot\left[\# \mathbf{S}^{\mathbf{j}}\right]_{k \ell}}{\sum_{\ell=1}^{\ell_{\max }} \sum_{\substack{q=1, \ldots,|\mathcal{P}| \\ l_{q}=\ell}} \omega_{\ell} b_{k q}} .
$$

The weighted sum is used here due to its ability to attribute a key role to the effectiveness (i.e., the level of prevention) of the specific preventive actions performed by the worker, in order to carefully describe his/her level of caution toward risk $r_{k}$.

The level of caution $\tau_{i j}$ of worker $w_{j}$ with respect to task $t_{i}$ is defined as the ratio of the following two terms: 1) the Euclidean norm of the vector resulting from the Hadamard product (denoted with $\circ$ ) of vector $\boldsymbol{\rho}^{\mathbf{j}} \in[0,1]^{R_{i}}$ that contains the worker's levels of caution for each of the $R_{i}=\sum_{k=1}^{|\mathcal{R}|} e_{i k}$ risks of $t_{i}$, and vector $\mathbf{h}^{\mathbf{i}} \in(0,1]^{R_{i}}$ containing the hazardousness of the risks of task $\left.t_{i} ; 2\right)$ the Euclidean norm of vector 1 expressing the highest level of caution for task $t_{i}$, i.e., $\|\mathbf{1}\|=\sqrt{R_{i}}$. Hence,

$$
\tau_{i j}=\frac{\left\|\mathbf{h}^{\mathbf{i}} \circ \boldsymbol{\rho}^{\mathbf{j}}\right\|}{\sqrt{R_{i}}}=\frac{1}{\sqrt{R_{i}}} \sqrt{\sum_{k=1}^{R_{i}}\left(h_{k} \rho_{k}^{j}\right)^{2}} .
$$

The Euclidean norm is used in (12) to straightforwardly determine the deviation of the worker's strategy from the safest strategy adopted to interact with the risk, i.e., all its preventive actions performed. Note that $\rho_{k}^{j} \equiv \rho_{k j}$.

\section{Human factors}

1) Task-independent human factors and score functions: Let $\mathcal{F}^{\prime}=\left\{f_{1}, \ldots, f_{\left|\mathcal{F}^{\prime}\right|}\right\} \subset \mathcal{F}$ be the set of task-independent human factors, i.e., personal and psychological factors. Each worker is characterized by a set of values, each one associated with a task-independent human factor in $\mathcal{F}^{\prime}$. Formally, 
let $\mathcal{F}_{+}^{\prime}=\left\{f_{1}^{+}, \ldots, f_{\left|\mathcal{F}_{+}^{\prime}\right|}^{+}\right\}$and $\mathcal{F}_{-}^{\prime}=\left\{f_{1}^{-}, \ldots, f_{\left|\mathcal{F}_{-}^{\prime}\right|}^{-}\right\}$, where $\mathcal{F}_{+}^{\prime} \cup \mathcal{F}_{-}^{\prime}=\mathcal{F}^{\prime}$ and $\mathcal{F}_{+}^{\prime} \cap \mathcal{F}_{-}^{\prime}=\emptyset$, be the set of task-independent human factors whose values are directly proportional to increasing levels of risk perception (or to decreasing levels of risk propensity), and the set of task-independent human factors whose values are directly proportional to decreasing levels of risk perception (or to increasing levels of risk propensity), respectively. Let $\mathcal{D}_{u}^{+}$ and $\mathcal{D}_{v}^{-}$indicate, respectively, the domain of $f_{u}^{+}$and $f_{v}^{-}$, where $u \in\left\{1, \ldots,\left|\mathcal{F}_{+}^{\prime}\right|\right\}$ and $v \in\left\{1, \ldots,\left|\mathcal{F}_{-}^{\prime}\right|\right\}$. Typical domains are, for example, $\mathbb{N}$ for the number of past industrial injuries, \{very low, low, medium, high, very high\} for the perceived level of control over a specific task, and so on.

The task-independent human factors status of worker $w_{j}$ is represented by a vector $\mathbf{f}^{\mathbf{j}} \in \mathcal{D}_{1}^{+} \times \cdots \times \mathcal{D}_{\left|\mathcal{F}_{+}^{\prime}\right|} \times \mathcal{D}_{1}^{-} \times$ $\cdots \times \mathcal{D}_{\left|\mathcal{F}_{-}^{\prime}\right|}^{-}$. Each element of $\mathrm{f}^{\mathrm{j}}$ contains the value of the corresponding human factor for $w_{j}$.

More rigorously, let $f_{u, j}^{+} \in \mathcal{D}_{u}^{+}$and $f_{v, j}^{-} \in \mathcal{D}_{v}^{-}$be the value of the human factor $f_{u}^{+} \in \mathcal{F}_{+}^{\prime}$ and $f_{v}^{-} \in \mathcal{F}_{-}^{\prime}$ for $w_{j}$, respectively. Two sets of linear score functions are defined to assign human factor scores in terms of sensitivity to risk to the values of each human factor. Linearity stems from the correlation between the value of the human factors and its impact on risk perception and risk propensity, referred to in Section V-A. In particular, the first set $\left\{\Gamma_{u}^{+}\left(f_{u}^{+}\right), f_{u}^{+} \in \mathcal{D}_{u}^{+}, \forall u=\right.$ $\left.1, \ldots,\left|\mathcal{F}_{+}^{\prime}\right|\right\}$ contains score functions $\Gamma_{u}^{+}: \mathcal{D}_{u}^{+} \rightarrow[0,1]$ such that $f_{u, j}^{+} \mapsto \Gamma_{u}^{+}\left(f_{u, j}^{+}\right)$and $\Gamma_{u}^{+}\left(f_{u, j}^{+}\right) \propto f_{u, j}^{+}$. The other set $\left\{\Gamma_{v}^{-}\left(f_{v}^{-}\right), f_{v}^{-} \in \mathcal{D}_{v}^{-}, \forall v=1, \ldots,\left|\mathcal{F}_{-}^{\prime}\right|\right\}$ contains score functions $\Gamma_{v}^{-}: \mathcal{D}_{v}^{-} \rightarrow[0,1]$ such that $f_{v, j}^{-} \mapsto \Gamma_{v}^{-}\left(f_{v, j}^{-}\right)$ and $\Gamma_{v}^{-}\left(f_{v, j}^{-}\right) \propto-f_{v, j}^{-}$. Score functions can be defined with the help of an expert in risk assessment in order to map the values of the domain of each task-independent human factor into an appropriate human factor score in $[0,1]$ in terms of sensitivity to risk. Note that values close to 1 mean a higher risk perception (better), while values close to 0 mean a lower risk perception (worse). Figure 4 shows an example of a score function for the task-independent human factor "perceived level of work control" (i.e., the extent to which individuals believe they can control the task they perform) whose values are directly proportional to decreasing levels of risk perception.

The global task-independent human factors score $\varphi_{j} \in$ $[0,1]$ of $w_{j}$ (hereafter, global task-independent score) is defined as

$$
\varphi_{j}=\left|\mathcal{F}^{\prime}\right| \cdot\left(\sum_{u=1}^{\left|\mathcal{F}_{+}^{\prime}\right|} \frac{1}{\Gamma_{u}^{+}\left(f_{u, j}^{+}\right)}+\sum_{v=1}^{\left|\mathcal{F}_{-}^{\prime}\right|} \frac{1}{\Gamma_{v}^{-}\left(f_{v, j}^{-}\right)}\right)^{-1},
$$

where the harmonic mean is used because of its ability to mitigate (intensify) the impact of large (small) outliers.

2) The expertise human factor: To quantify how experienced a worker is in performing a task, the task-related $e x$ pertise human factor is considered. This serves as a constraint in the model so that each worker has the minimum expertise required for the task assigned. The expertise human factor is modeled as a function of a worker's ability to perform a task and how recently the worker last executed that task.

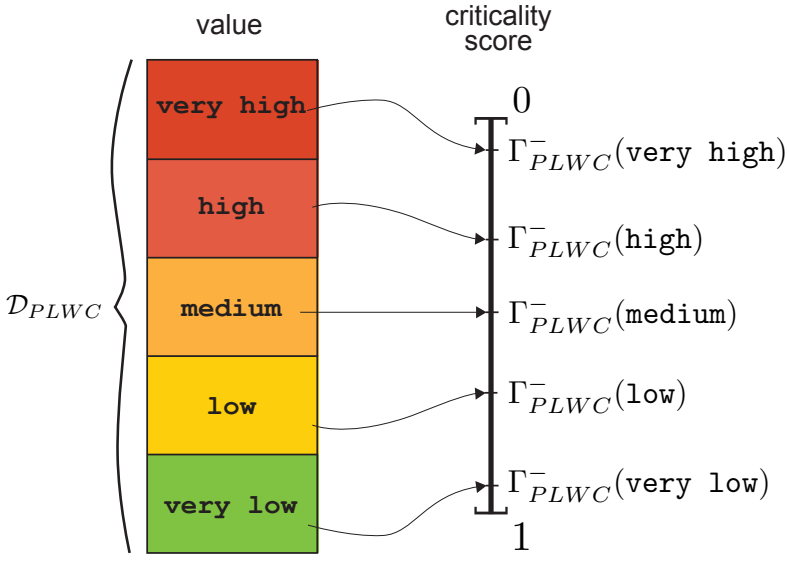

Fig. 4. Mapping of a score function $\Gamma_{P L W C}^{-}$for the task-independent human factor "perceived level of work control" (PLWC). Low values of the domain $\mathcal{D}_{P L W C}$ of PLWC are mapped into high scores and vice versa.

Formally, consider a worker $w_{j}$ and a task $t_{i}$. Let $a_{i j} \in$ $(0,1]$ be the ability of $w_{j}$ in performing $t_{i}$. Let $\mathcal{P}_{i, j}$ be the set of past jobs wherein $w_{j}$ performed $t_{i}$, and let $p \in \mathcal{P}_{i, j}$ be one of these past jobs. Let $D_{j, p}^{S T A R T}$ and $D_{j, p}^{E N D}$ be the date on which worker $w_{j}$ began and stopped his/her previous employment $p$, respectively.

Let the function days (date 1, date 2 ) return the number of days between date 1 and date2, and let NOW represent the current date. In addition, let the maximum of a set of dates be the most recent date. The score of the expertise human factor of $w_{j}$ in performing $t_{i}$ is

$$
z_{i j}=a_{i j}+\frac{\omega_{P A S T} \sum_{p \in \mathcal{P}_{i, j}} \operatorname{days}\left(D_{j, p}^{S T A R T}, D_{j, p}^{E N D}\right)}{\omega_{I D L E} \cdot \operatorname{days}\left(\max _{p \in \mathcal{P}_{i, j}} D_{j, p}^{E N D}, \text { NOW }\right)},
$$

where $a_{i j}$ is expressed by an expert who evaluates the ability of $w_{j}$ in performing $t_{i}$, through, for example, a practical test performed in a risk-free context. Weights $\omega_{P A S T}$ and $\omega_{I D L E}$, such that $\omega_{P A S T}+\omega_{I D L E}=1$, are intended to be expressed by the management based on the importance given to the global duration of the past jobs concerning a given task, and how long it has been since the worker performed that task for the last time, respectively. As an alternative, the management may compute $z_{i j}$ just considering the worker's past jobs, thus neglecting the term $a_{i j}$ in (14). The possible current job $p^{\star}$ of $w_{j}$ is considered as a past job with $D_{j, p^{\star}}^{E N D}=$ NOW. Note that if worker $w_{j}$ is currently performing task $t_{i}$, the denominator of (14) would be equal to zero, since days (NOW, NOW) $=0$, and $z_{i j} \rightarrow+\infty$, which would make no sense. In this case, days() is assumed to return $\omega_{P A S T} / \omega_{I D L E}$, and the contribution of $\omega_{P A S T}$ and $\omega_{I D L E}$ is canceled because whenever a worker is currently performing a given task, what actually matters for the calculation of $z_{i j}$ is just the number of days until the current day that the worker has been performing the task.

Equation (14) stems from the following consideration. The total time a worker spends performing a particular task leads him/her to achieve a certain level of expertise for that task, regardless of the time intervals (in the past) when the worker 
was not performing it. Given the specificity of the tasks of a manufacturing company, which are composed of specific sequences of actions and precautions to be taken at any time, if a worker performed a task in the past, but he/she is no longer performing the task, a decrease in expertise has certainly occurred, which is intuitively proportional to the time elapsed since the last time he/she performed the task. For manufacturing tasks, it is thus reasonable to assume that the longer the time since a worker last performed a task, the less likely he/she will precisely remember the steps involved, and their particular sequence of actions. We therefore considered the decrease in expertise as linearly dependent on the time elapsed since the last time the worker performed the task. More complex (and more accurate) formulations could be investigated, but this is not within the main scope of this paper.

3) The dislike for task human factor: The task-related dislike for task human factor is used to formalize an objective function, as will be explained in Section VII-B.

\section{Worker's carefulness}

The carefulness of a worker with respect to a given task blends the worker's level of caution for the task (i.e., the safety level of his/her behavior when executing the task) and his/her human factors configuration to measure how much the worker's careful forethought is able to prevent the injuries caused by the risks to which he/she is exposed while performing that task. Note that carefulness differs from caution as caution uniquely stems from the worker's behavior, no matter his/her human factors configuration.

Formally, we define the carefulness of worker $w_{j}$ with respect to task $t_{i}$ as

$$
\chi_{i j}=\gamma_{i j} \tau_{i j},
$$

where $\tau_{i j}$ is the level of caution of $w_{j}$ with respect to $t_{i}$ as defined in (12), and the coefficient $\gamma_{i j}$ is formally defined in (16). In more detail, $\gamma_{i j}$ depends on the problem dealt with: personnel reassignment or personnel recruitment. Personnel reassignment aims to optimize the objectives by changing some of the current employee-task associations. Instead, starting from a set of candidates, personnel recruitment aims to select the most appropriate people to hire for the vacant positions in a company.

For reassignment problems, the quantity $\left|\varphi_{j}-\eta_{i}\right|$ in (16) measures the closeness of the worker's global task-independent score $\varphi_{j}$ to the hazardousness $\eta_{i}$ of the task: the higher the closeness, the less the caution is penalized by the global taskindependent score. This essentially means that, given a task $t_{i}$, using the term $\left(1-\left|\varphi_{j}-\eta_{i}\right|\right)$ in (16), a worker $w_{j}$ is assumed to be more suitable to perform a task, the closer his/her global task-independent score $\varphi_{j}$ is to the hazardousness $\eta_{i}$ of the task. The lower the worker's global task-independent score with respect to the hazardousness of a task, the more likely the worker is to get hurt, if assigned to that task with no risk awareness training. Conversely, the higher the worker's global task-independent score with respect to the hazardousness of a task, the less the worker's performance is properly exploited regarding the task-independent human factors influencing risk perception, i.e., the worker could be safely assigned to riskier tasks. Figure 5 shows a plot of $\gamma_{i j}$ versus $\left(\varphi_{j}-\eta_{i}\right)$.

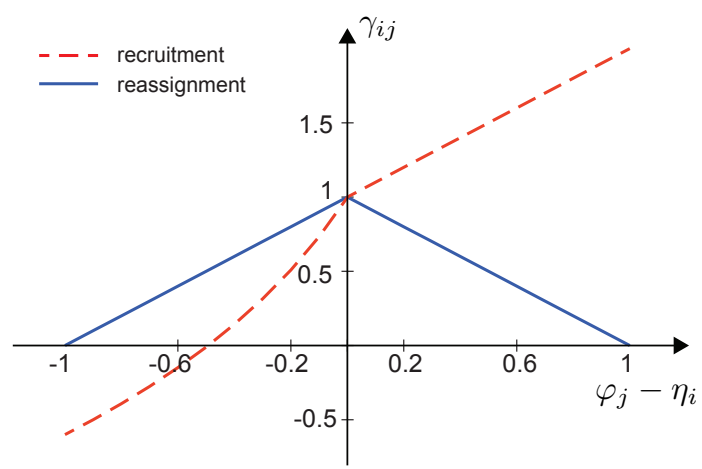

Fig. 5. Plot of $\gamma_{i j}$ versus $\left(\varphi_{j}-\eta_{i}\right)$ for reassignment (solid line) and recruitment (dashed line).

For recruitment problems, $\gamma_{i j}$ has a different shape depending on the sign of $\varphi_{j}-\eta_{i}$. In detail, if $\varphi_{j}-\eta_{i} \geq 0$, that is, the worker's global task-independent score is higher than, or equal to, the task's hazardousness, the worker's level of caution $\tau_{i j}$ in (15) is multiplied by a positive term (i.e., $\gamma_{i j}=1+\varphi_{j}-\eta_{i}$ ) which is linearly dependent on $\varphi_{j}-\eta_{i}$. This means that, given $\tau_{i j}$, the better the worker's performance regarding the task-independent human factors, the more $\tau_{i j}$ is increased. On the other hand, if $\varphi_{j}-\eta_{i}<0$, the term $\gamma_{i j}$ acts as a penalty coefficient for the worker's caution $\tau_{i j}$ in (15). In particular, a logarithmic penalty is considered here, i.e., $\gamma_{i j}=1-\frac{\ln \left(1-2\left(\varphi_{j}-\eta_{i}\right)\right)}{\ln (2)}$. The logarithm is chosen since the caution of a worker with a global task-independent score lower than the task hazardousness, i.e., $\varphi_{j}-\eta_{i}=-\Delta$, with $\Delta \in[0,1)$, needs to be penalized more drastically than it would be augmented for the same difference, but opposite in sign, i.e., if $\varphi_{j}-\eta_{i}=\Delta$. This is justified by the fact that, when $\varphi_{j}-\eta_{i}=-\Delta$, assigning a worker $w_{j}$ to task $t_{i}$ is considered increasingly unsafe as $|\Delta|$ increases, because of the worker's lower innate ability to perceive the risks

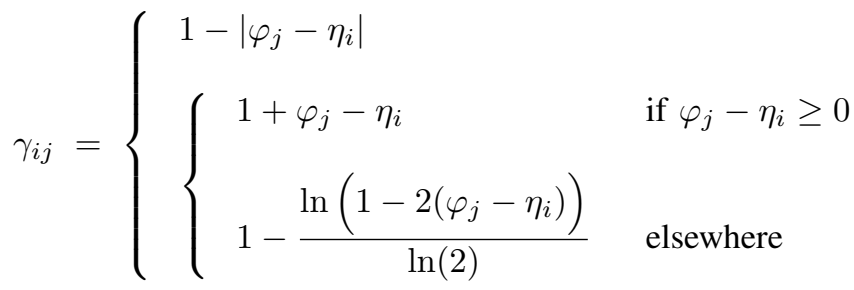

for personnel recruitment problems 
and, therefore, the danger to which he/she is exposed, with a consequent increase in the probability of the risk materializing and/or having a negative impact. The values of the logarithmic penalty coefficients (i.e., $\frac{1}{\ln (2)}$ and 2 ) were chosen to achieve the desired shape of the penalty function for $\varphi_{j}-\eta_{i} \in[-1,0]$. In particular, for $\varphi_{j}-\eta_{i} \in[-1,-0.5]$ the penalty is negative, which means that the corresponding worker-task assignment is considered even more unsafe.

\section{OPTIMIZATION PROBLEM}

\section{A. Objective functions}

The aim of the proposed optimization model is to optimize three objectives: COST, DISLIKE and CAREFULNESS.

Let $x_{i j} \in\{0,1\}$ be a decision variable such that

$$
x_{i j}= \begin{cases}1 & \text { if task } t_{i} \text { is assigned to worker } w_{j} \\ 0 & \text { otherwise }\end{cases}
$$

where $i \in\{1, \ldots,|\mathcal{T}|\}$ and $j \in\{1, \ldots,|\mathcal{W}|\}$. The vector $\mathbf{x} \in$ $\{0,1\}^{|\mathcal{T}| \times|\mathcal{W}|}$ represents a personnel assignment and contains the decision variables $x_{i j}$ in lexicographic order, as elements.

Consider the cost $c_{i j}$ of assigning task $t_{i}$ to worker $w_{j}$, which may be conceived as the amount of money a company has to pay for $w_{j}$ if assigned to $t_{i}$ (i.e., the employment cost together with the cost of training). As in the classic version of the job assignment problem, one of the objectives is to minimize the global cost, here defined as:

$$
\begin{gathered}
\operatorname{COST}(\mathbf{x})=\sum_{i=1}^{|\mathcal{T}|} \sum_{j=1}^{|\mathcal{W}|} c_{i j} x_{i j}= \\
\sum_{i=1}^{|\mathcal{T}||\mathcal{W}|}\left(\sum_{j=1}^{E M P L O Y M E N T}+c_{i j}^{T R A I N I N G}\right) x_{i j} .
\end{gathered}
$$

In (17), $c_{j}^{E M P L O Y M E N T}$ is the employment cost of worker $w_{j}$, i.e., the actual amount the employer pays for the employee's salary and benefits. The employment cost includes the salary, commissions, taxes such as social security and insurance, insurance premiums and pension schemes, as well as the cost of any other fringe benefits. The other term, i.e., $c_{i j}^{T R A I N I N G}$, is the cost of training $w_{j}$ if assigned to $t_{i}$.

In the proposed model, the workers' global dislike for the task assigned is also minimized. Let $d_{i j} \in\left\{0, \ldots, d_{\max }\right\}$ be the value of the dislike for task human factor expressed by worker $w_{j}$ for each task $t_{i}$; the greater $d_{i j}$ the less $w_{j}$ likes $t_{i}$. Global dislike represents the workers' overall aversion to a personnel assignment $\mathbf{x}$, and is defined as:

$$
\operatorname{DISLIKE}(\mathbf{x})=\sum_{i=1}^{|\mathcal{T}|} \sum_{j=1}^{|\mathcal{W}|} d_{i j} x_{i j}
$$

Finally, global carefulness is maximized. The global carefulness of a personnel assignment $\mathrm{x}$ measures the extent to which riskier tasks are assigned to workers with higher levels of carefulness, and vice versa. More formally, the global carefulness function is defined as

CAREFULNESS $(\mathbf{x})=\sum_{i=1}^{|\mathcal{T}|} \sum_{j=1}^{|\mathcal{W}|} \chi_{i j} x_{i j}=\sum_{i=1}^{|\mathcal{T}|} \sum_{j=1}^{|\mathcal{W}|}\left(\gamma_{i j} \tau_{i j}\right) x_{i j}$, where $\chi_{i j}$ is the carefulness of worker $w_{j}$ with respect to task $t_{i}$ (see (15)), $\gamma_{i j}$ is the penalty term formalized in (16), and $\tau_{i j}$ is the worker's level of caution with respect to task $t_{i}$ (see (12)).

\section{B. Problem formulation}

Let us consider a set of tasks $\mathcal{T}$ and a set of workers $\mathcal{W}$, where, in general, $|\mathcal{W}| \lesseqgtr|\mathcal{T}|$. Assume that each task can be assigned to one worker and vice versa. Also, suppose that each worker can perform any task.

As stated in Section VII-A, global cost and global dislike are minimized, while global carefulness is maximized. Maximizing the global carefulness corresponds to minimizing its opposite, i.e., $-\sum_{i=1}^{|\mathcal{T}|} \sum_{j=1}^{|\mathcal{W}|}\left(\gamma_{i j} \tau_{i j}\right) x_{i j}$. Hence, the proposed multi-objective formulation of personnel assignment is modeled as the following minimization problem, using the notation and concepts introduced here and in Section VI:

$$
\begin{gathered}
\underset{\mathbf{x}}{\operatorname{Minimize}} \mathbf{f}(\mathbf{x})=[\operatorname{COST}(\mathbf{x}), \operatorname{DISLIKE}(\mathbf{x}), \\
{\left[\sum_{i=1}^{|\mathcal{T}|} \sum_{j=1}^{|\mathcal{W}|}\left(c_{j}^{E M P L O Y M E N T}+c_{i j}^{T R A I N I N G}\right) x_{i j},\right.} \\
\left.\sum_{i=1}^{|\mathcal{T}|} \sum_{j=1}^{|\mathcal{W}|} d_{i j} x_{i j},-\sum_{i=1}^{|\mathcal{T}|} \sum_{j=1}^{|\mathcal{W}|}\left(\gamma_{i j} \tau_{i j}\right) x_{i j}\right]
\end{gathered}
$$

subject to:

$$
\begin{gathered}
\left\{\begin{array}{c}
\sum_{i=1}^{|\mathcal{T}|} x_{i j}=1, \quad \forall j=1, \ldots,|\mathcal{W}| \quad \text { reassignment } \\
\sum_{i=1}^{|\mathcal{T}|} x_{i j} \leq 1, \quad \forall j=1, \ldots,|\mathcal{W}| \quad \text { recruitment }
\end{array}\right. \\
\sum_{j=1}^{|\mathcal{W}|} x_{i j}=1, \quad \forall i=1, \ldots,|\mathcal{T}| \\
z_{i j} x_{i j} \geq z_{i}^{\text {min }}, \quad \forall i=1, \ldots,|\mathcal{T}|, \eta_{i} \geq \eta^{\text {max }}, \forall j=1, \ldots,|\mathcal{W}| \\
(20 \mathrm{~d})
\end{gathered}
$$

Equation (20a) is the vector-valued objective function $\mathbf{f}(\mathbf{x}):\{0,1\}^{|\mathcal{T}| \times|\mathcal{W}|} \rightarrow \mathbb{R}^{+} \times\left[0, d_{\max }|\mathcal{T}|\right] \times$ $\left[-2|\mathcal{T}|,\left(1-\frac{\ln (3)}{\ln (2)}\right)|\mathcal{T}|\right]$ whose components are the global cost, the global dislike for the task assigned, and the opposite of the global carefulness for a personnel assignment $\mathbf{x} \in\{0,1\}^{|\mathcal{T}| \times|\mathcal{W}|}$. Constraints (20b), one for each worker, are problem-dependent; in personnel reassignment, they ensure each worker is (re)assigned to one task; in personnel recruitment, because of the imbalance of workers and tasks, a worker may not be assigned to a task. Constraints (20c), one for each task, force a task to be assigned to exactly one worker. Constraints (20d) express the minimum expertise $z_{i}^{\text {min }}$ required to perform each safety-critical task $t_{i}$, i.e., those tasks with a task hazardousness $\eta_{i}$ greater than a problem dependent critical threshold $\eta^{\max } \in(0,1]$. Finally, (20e) is the integer constraint. 


\section{RESOLUTION METHODOLOGY}

\section{A. Description and motivation}

The optimization problem (20a)-(20e) is solved with a mixed EMO-MCDM methodology.

In particular, weights are first assigned to the objectives using a fuzzy version of AHP. AHP is used since pairwise comparison makes it easier for the management to prioritize. This is because objectives are taken into account two at a time, which is close to the way people reason when ranking multiple objectives. Also, AHP has particular advantages when important elements of the decision are difficult to quantify or compare [30]. This is the case for carefulness, which is hard to correctly quantify for a manager because it is a new measure, which is dimensionless and intrinsically correlated with the level of safety in the workplace. It would thus be hard to express, for example, marginal rates of substitutions to derive priorities by analyzing the related indifference curves. Finally, to enable the management to express priorities realistically, vagueness and uncertainty are considered here using triangular fuzzy numbers, with an index of optimism for defuzzification [31], [32]. More accurate defuzzification techniques are possible, but this is not the main focus of the paper.

The NSGA-II algorithm is used in the resolution methodology to generate an approximation of the Pareto front. An evolutionary technique is chosen because, compared to weighting methods, it can generate a continuous and more homogeneous approximation of every zone of the Pareto front [33], so as to obtain a much wider choice of Pareto optimal solutions that are able to meet the decision maker's preferences more accurately.

TOPSIS automatically selects the solution to the problem within the last front by picking the Pareto-optimal solution representing the nearest compromise to the decision maker's preferences. TOPSIS is used because of its simplicity and ability to deal with the large amount of alternatives in the decision making process [34].

\section{B. Pseudocode}

The pseudocode in Algorithm 1 summarizes the optimization methodology. In the code snippet, four procedures are called. The first one, i.e., FUZZY_AHP $(\tilde{\mathbf{O}}, \zeta)$, takes, as arguments, the pairwise comparison matrix

$$
\tilde{\mathbf{O}}=\begin{gathered}
c \\
d \\
\chi \\
\chi
\end{gathered}\left(\begin{array}{ccc}
1 & \tilde{o}_{12} & \tilde{o}_{13} \\
\tilde{o}_{12}^{-1} & 1 & \tilde{o}_{23} \\
\tilde{o}_{13}^{-1} & \tilde{o}_{23}^{-1} & 1
\end{array}\right),
$$

where each element $\tilde{o}_{i j}$ is a TFN with the meanings defined in Table I, and an index of optimism $\zeta \in[0,1]$. FUZZY_AHP ( $\tilde{\mathbf{O}}$, $\zeta)$ returns the weights $\boldsymbol{\omega}$ of the objectives. The second procedure is INITIALIZE (pop_size, $|\mathcal{W}|,|\mathcal{T}|, \mathbf{A}, \mathbf{b}$, $\left.\mathbf{A}_{\mathbf{e q}}, \mathbf{b}_{\mathbf{e q}}, \mathbf{c}, \mathbf{d}, \chi\right)$. This procedure generates and returns the initial population $\mathbf{X}$ of the genetic algorithm, where each individual is a feasible personnel assignment. The parameters of the procedure are, in order, the number of individuals of the population pop_size, the number of workers $|\mathcal{W}|$, the number of tasks $|\mathcal{T}|$, the inequality and equality constraints, respectively, in matrix notation, i.e., $\mathbf{A}, \mathbf{b}$ and $\mathbf{A}_{\mathbf{e q}}, \mathbf{b}_{\mathbf{e q}}$. Also, $\mathbf{c}, \mathbf{d}$ and $\chi$, containing, in the order, all the worker-task values of cost, dislike and carefulness, are used to generate individuals with appropriate values for the objectives. Procedure NSGAII (X, A, b, $\mathbf{A}_{\mathbf{e q}}, \mathbf{b}_{\mathbf{e q}}$, cross_rate, mut_rate, $\mathbf{c}$, $\mathbf{d}, \chi)$ performs one iteration of the NSGA-II algorithm with crossover rate cross_rate and mutation rate mut_rate. Pareto dominance is evaluated according to parameters $\mathbf{c}, \mathbf{d}$ and $\chi$. Finally, procedure TOPSIS $(\mathbf{X}, \boldsymbol{\omega})$ selects the best solution $\mathrm{x}^{\mathrm{BEST}}$ from the final population $\mathbf{X}$ according to the weights in $\boldsymbol{\omega}$, both taken as arguments.

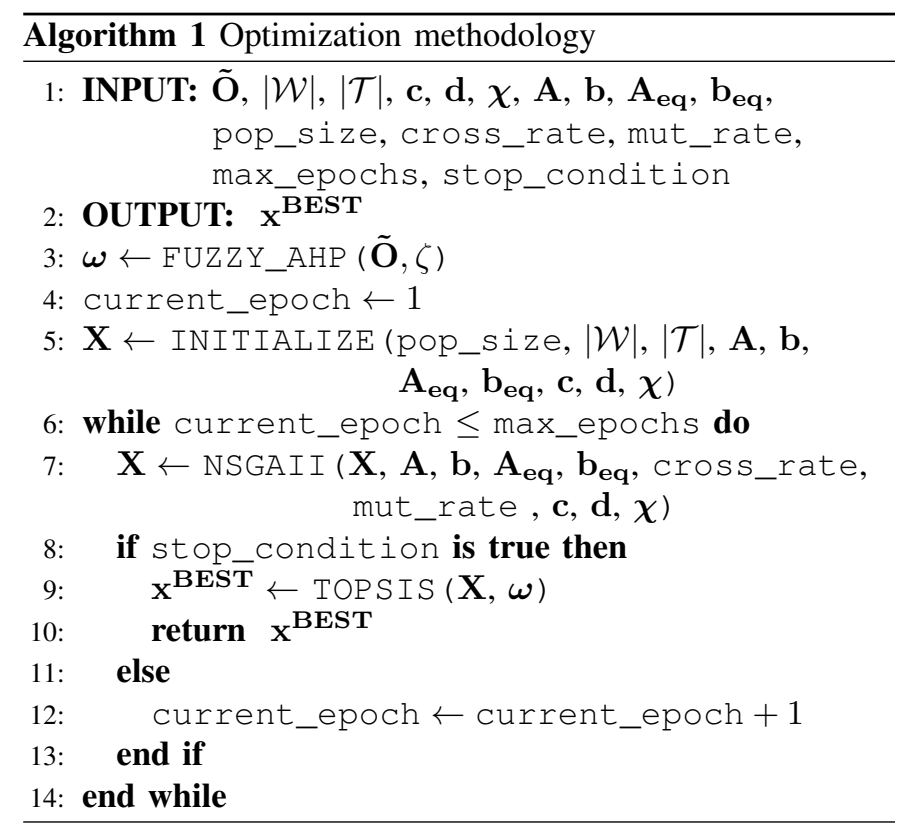

\section{EXPERIMENTS AND DISCUSSION}

This section presents the results obtained by applying the proposed framework to two different real-world personnel assignment problems, namely, personnel recruitment, and personnel reassignment, in small manufacturing companies producing shoes, which voluntarily accepted to take part in the experiments.

To the best of the authors' knowledge, this is the first work including the workers' sensitivity to risk in a personnel assignment problem, and it was thus not possible to include any comparisons with other existing approaches. The discussion is therefore based on comparing the assignments obtained with the proposed framework to those suggested (or currently adopted) by the management of the factories involved. In addition, data related to optimization times are not included since the problems have no strict time constraint.

\section{A. Dataset and implementation}

The optimization framework was implemented in $\mathrm{Java}^{\mathrm{TM}}$ and MATLAB ${ }^{\circledR}$. A web application was also implemented in Java $^{\mathrm{TM}}$ EE and MySQL, and deployed on a web server. The web application implements a website containing a questionnaire, as a multiple choice test. Workers (or applicants) from various shoe factories remotely and anonymously filled out the 
questionnaire, thus providing us with the data needed for the experiments. The questionnaire was aimed at:

- collecting data related to the task-independent human factors of Table II, in order to compute the worker's human factors scores and global task-independent score $\varphi_{j}$ (see (13)). Psychological factors, i.e., anxiety, worry and self-esteem were measured with the Zung test [35], Penn State Worry Questionnaire [36], and Rosenberg test [37], respectively;

- collecting data related to the task-related human factors of Table II. In particular, the values $d_{i j}$ of the dislike for task human factor were expressed by using the scale \{very low, low, medium, high, very high . These values were then mapped uniformly into $[0,1]$. To quantify the expertise human factor for each task (see (14)), data related to each past job duration are required. The ability $a_{i j}$ is evaluated with a risk-free practical test in the factory;

- for each task $t_{i}$, acquiring the worker's strategy for each risk $r_{k}$ of task $t_{i}$, i.e., each risk such that $e_{i k}=1$. The strategy is composed of the preventive actions $p_{q}$ (such that $b_{k q}=1$ ) chosen by the worker from a finite set of proposed preventive actions derived from the behavior matrix B (see (7)). The chosen actions determine the elements equal to 1 in the rows $\mathbf{s}_{\mathbf{k}}^{\mathbf{j}}$ of the worker's global strategy matrix $\mathbf{S}^{\mathbf{j}}$ (see (8))

Due to limited space, the questionnaire is not reported.

The overall collected dataset consisted of 131 interviews. A total of 100 interviews related to a recruitment process were used to test the proposed framework regarding a real personnel recruitment problem (REC). The results are discussed in Section IX-D.

The remaining interviews, related to three different shoe factories with 8,10 , and 13 workers, respectively, were used in relation to personnel reassignment, in order to show the significant increase in global carefulness (and thereby the contribution of the proposed approach to making risks less likely and/or less dangerous) which is achievable by simply reassigning (part of the) tasks. The results of this second experiment are discussed, as REA1, REA2, and REA3, in Section IX-E.

\section{B. Genetic encoding}

In this work an integer-valued encoding was used. A personnel assignment is represented by a chromosome with as many genes as the number of tasks: the value of a gene is an integer identifier of the worker assigned to the task associated with the gene. Different genes must contain different values.

\section{Parameters of NSGA-II}

Simulations were carried out to find the best values for the parameters of NSGA-II. More precisely, three population sizes, i.e., $\{250,300,350\}$, three crossover probabilities, i.e., $\{0.25,0.55,0.85\}$ and three mutation probabilities, i.e., $\{0.01,0.05,0.09\}$ were first chosen based on heuristic considerations, also considering that mutation probability is typically one order of magnitude lower than crossover probability. Population sizes were heuristically selected to obtain a fairly uniform approximation of the Pareto front.

By combining these values, 27 configurations of parameters were obtained. The maximum number of iterations was kept to 1000 . For each configuration, twenty runs were performed, starting from different initial populations. At the end of each run, the best Pareto-optimal solution was selected by TOPSIS, thanks to the weights determined with F-AHP. Finally, the average global cost, dislike and carefulness of all the solutions obtained by every run of the considered configuration of parameters were computed. The Student's $t$-test was used to validate the statistical significance, where the null hypothesis $\mathcal{H}_{0}$ assumed that the difference in the mean values is due to chance. A total of 339 comparisons (i.e., $\frac{(27-1)^{2}}{2}+1$ ) were carried out to compute Student's $t$-test values for each objective. The critical value $t^{C R I T I C A L}$ for $95 \%$ confidence was chosen. Hence, considering two compared configurations of parameters, $\mathcal{H}_{0}$ is rejected if their $t$ value exceeds $t^{C R I T I C A L}$. The configuration resulting in the greatest number of $\mathcal{H}_{0}$ rejections was chosen since it is statistically better than any other configuration.

\section{Personnel recruitment (REC)}

1) Description of the problem: Personnel recruitment is carried out by companies to select the most suitable worker to assign to each vacant position. Usually, an expert evaluates how experienced a candidate is in performing a task by analyzing his/her past jobs and their duration, and evaluating his/her ability in performing the task through an appropriate, risk-free practical test. The higher the candidate's ability in performing a given task, the lower the cost of assigning the candidate to that task, thanks to the lower cost of training.

2) Personnel recruitment in the company involved: Personnel recruitment consists of the following steps, in which candidates:

- provide a curriculum vitae;

- perform some practical tests;

- have an oral interview.

A suitability degree for each task is eventually assigned to each candidate based on the analysis of the curriculum vitae, the outcome of the practical tests and the interview. In addition, the cost is taken into account, in order to minimize the capital allocated for the recruitment of new employees. The management considers the cost of assigning a worker to a task as dependent on his/her work seniority and level of dexterity in performing the task.

3) Limitations: Although the footwear industry is characterized by serious risks, including crushing injuries, intoxication, amputation, falls and burns, in the factories considered, risk management is carried out exclusively by assigning more dangerous tasks to candidates with a higher number of years of experience. This is an extremely inefficient risk management approach, mainly because a person's expertise is only one of the human factors influencing risk perception and propensity. All the other factors introduced in Section V-A are ignored by the management. Moreover, by merely considering the 
worker's expertise for the assignment of dangerous tasks, the worker's behavior when dealing with risks is in no way taken into consideration, leading to inevitable inefficiencies.

4) Proposed technique for personnel recruitment: Using the proposed framework, the management was first asked to fill in a PCM for the objectives (i.e., cost, dislike and carefulness) by means of judgments expressed in terms of a fuzzyfied Saaty scale of preference, with the meanings shown in Table I. The weights of the objectives were derived through F-AHP and are shown in the first row of Table III.

An expert in risk assessment configured the score functions needed to obtain the global task-independent score (see (13)). This expert classified the preventive actions into three levels of prevention (i.e., low, medium, high) associated with exponentially increasing weights (the terms $\omega_{\ell}$ in (11)).

The optimization framework was applied to a personnel recruitment process (REC) carried out by a footwear company. The company had to select 10 new workers to be assigned to 10 tasks. The management considered a total of 100 applicants for the vacant positions of the company. A candidate generally applies for a single position (i.e., task), thus the expertise in other tasks is considered to be zero. A practical test was therefore required just for the position each applicant applied for.

Data related to each candidate's human factors and strategy toward each risk were gathered using the questionnaire described in Section IX-A, in order to obtain each candidate's human factors scores and his/her level of caution toward each risk. The level of caution with respect to each task and the global task-independent score of each candidate were first computed according to (12) and (13), respectively. Then, the carefulness of each candidate with respect to each task was computed according to (15). The Pareto front was generated by means of NSGA-II using the parameters in Table IV.

The best solution to the problem is reported in Table V. The table is organized into horizontal and vertical blocks. The two vertical blocks (labeled "Tasks" and "Objectives") refer to the tasks to be assigned (indicated as "T1", "T2", and so on) and the values of the objective functions, respectively. Each horizontal block is related to a specific scenario (REC, REA1, REA2, or REA3) and is split into two rows labeled as "Suggested" and "Proposed" for REC, and "Current" and "Proposed" for REA1, REA2 and REA3. Consider a horizontal block. Each cell in the first vertical block contains the identifier
TABLE III

WEIGHTS OF THE OBJECTIVES FOR THE PERSONNEL RECRUITMENT AND REASSIGNMENT PROBLEMS

\begin{tabular}{|c|c|c|c|}
\cline { 2 - 4 } \multicolumn{1}{c|}{} & COST & DISLIKE & CAREFULNESS \\
\hline REC & 0.4917 & 0.1024 & 0.4059 \\
\hline REA1 & 0.3184 & 0.2107 & 0.4709 \\
\hline REA2 & 0.5824 & 0.0372 & 0.3804 \\
\hline REA3 & 0.0309 & 0.3866 & 0.5825 \\
\hline
\end{tabular}

TABLE IV

PARAMETERS USED FOR THE PERSONNEL RECRUITMENT (REC) AND THE PERSONNEL REASSIGNMENT PROBLEMS (REA1, REA2, REA3)

\begin{tabular}{|c|c|c|c|c|}
\cline { 2 - 5 } \multicolumn{1}{c|}{} & REC & REA1 & REA2 & REA3 \\
\hline Chromosome encoding & \multicolumn{4}{c|}{ Integer string } \\
\hline Selection method & \multicolumn{5}{c|}{ Binary tournament } \\
\hline Crossover operator & \multicolumn{5}{c|}{ Partially matched crossover } \\
\hline Mutation operator & \multicolumn{5}{c|}{ Swatation } \\
\hline Population size & 300 & 250 & 300 & 350 \\
\hline Crossover rate & 0.85 & 0.55 & 0.85 & 0.85 \\
\hline Mutation rate & 0.05 & 0.01 & 0.05 & 0.09 \\
\hline Max number of iterations & \multicolumn{4}{c|}{1000} \\
\hline
\end{tabular}

of the worker assigned to the corresponding task for the suggested/current (or proposed) personnel assignment; each cell in the second vertical block contains the value of the corresponding objective for the suggested/current (or proposed) personnel assignment.

Finally, the left hand side of Fig. 6 shows the scatter plot of the Pareto front viewed from two different perspectives, indicated as (a) and (b). In the plots, circles represent Paretooptimal personnel recruitments.

5) Discussion: Within REC, the aim is to improve the carefulness with a minimal increase in cost. This is evident from the weights of cost and carefulness in Table III, which are quite similar. Dislike, in this case, plays a marginal role, with a lower weight of 0.1024 .

Table $\mathrm{V}$ shows that the management suggested a recruitment with global carefulness equal to -2.731 . With the proposed recruitment, a $235.41 \%$ improvement in global carefulness is achieved. This is an outstanding improvement. The extent of safety in dealing with risk is highlighted by the fact that more than $70 \%$ of workers now (i.e., after our solution was

TABLE V

PERSONNEL ASSIGNMENTS WITH CORRESPONDING OBJECTIVES VALUES FOR THE EXPERIMENTED RECRUITMENT AND REASSIGNMENT PROBLEMS

\begin{tabular}{|c|c|c|c|c|c|c|c|c|c|c|c|c|c|c|c|c|c|}
\hline & \multicolumn{13}{|c|}{ Tasks } & \multicolumn{3}{|c|}{ Objectives } \\
\hline & & T1 & T2 & T3 & T4 & T5 & T6 & T7 & T8 & T9 & T10 & T11 & T12 & T13 & $\operatorname{COST}[€]$ & DISLIKE & CAREFULNESS \\
\hline \multirow{2}{*}{ REC } & Suggested & 24 & 77 & 51 & 59 & 86 & 12 & 47 & 2 & 90 & 72 & - & - & - & 22,490 & 9.54 & -2.731 \\
\hline & Proposed & 29 & 77 & 13 & 54 & 63 & 84 & 2 & 36 & 97 & 57 & - & - & - & 24,153 & 3.11 & 3.698 \\
\hline \multirow{2}{*}{ REA1 } & Current & 5 & 7 & 2 & 6 & 1 & 4 & 8 & 3 & - & - & - & - & - & 15,240 & 7.44 & 2.157 \\
\hline & Proposed & 3 & 7 & 2 & 6 & 1 & 4 & 5 & 8 & - & - & - & - & - & 17,200 & 2.45 & 3.847 \\
\hline \multirow{2}{*}{ REA2 } & Current & 2 & 9 & 4 & 6 & 3 & 10 & 1 & 5 & 8 & 7 & - & - & - & 20,496 & 1.18 & 3.021 \\
\hline & Proposed & 7 & 1 & 4 & 6 & 8 & 10 & 9 & 5 & 3 & 2 & - & - & - & 21,570 & 2.86 & 5.414 \\
\hline \multirow{2}{*}{ REA3 } & Current & 4 & 12 & 9 & 2 & 13 & 6 & 11 & 3 & 10 & 7 & 1 & 5 & 8 & 34,780 & 8.52 & 3.551 \\
\hline & Proposed & 4 & 10 & 9 & 2 & 13 & 8 & 11 & 1 & 3 & 7 & 12 & 5 & 6 & 41,577 & 2.96 & 6.695 \\
\hline
\end{tabular}




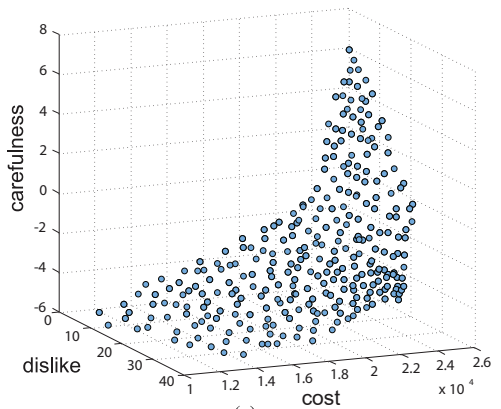

(a)

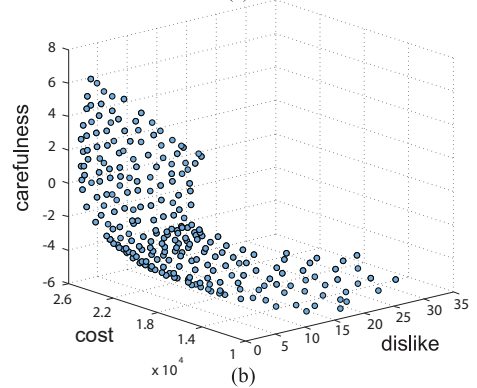

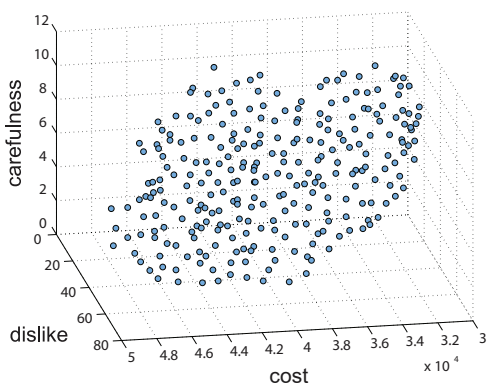

(c)

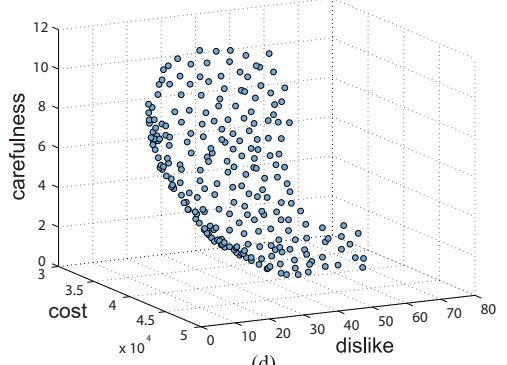

Fig. 6. Different views of the Pareto front of two representative scenarios: (a) and (b) are views of the Pareto front of the personnel recruitment experiment (REC); (c) and (d) show the Pareto front of the personnel reassignment related to the factory with 13 workers (REA3).

implemented) deal with the assigned task with a strategy exclusively composed of high-level preventive actions. The most unsafe strategy is composed of two medium-level actions and one low-level action for a worker assigned to a task with a hazardousness of 0.37. Data on the strategies are omitted here for reasons of space. Finally, the proposed recruitment brings the global dislike down to one-third of the global dislike of the assignment suggested by the management. There is no worker with a dislike for the task higher than medium, and just three workers express a medium dislike for the assigned task. Therefore, although global dislike is not one of the paramount objectives of REC, the proposed recruitment creates a workplace where workers are much more satisfied with the assigned tasks, with important positive consequences on both risk perception and the work climate. All these improvements are possible at just a $7.39 \%$ increase in the global cost.

Figure 7 shows a clustered column chart of the objectives values for suggested/current and proposed personnel assignments, for all the scenarios tested. Each cluster is composed of three bars representing, from left to right, the values of global cost, global dislike and global carefulness. With reference to REC, it is possible to compare the values of the objectives of the suggested and proposed recruitment, by referring to the first two clusters on the left.

\section{E. Personnel reassignment (REA1, REA2, REA3)}

1) Description of the problem: Personnel reassignment consists in reallocating the personnel of a company in order to obtain the improvement of some objective. The optimization model described in this paper was applied to three scenarios of personnel reassignment (REA1, REA2, and REA3), each related to a different footwear company. The following sec-

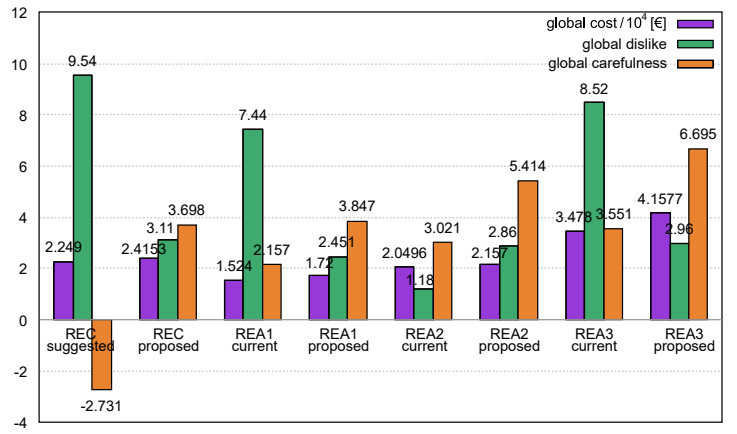

Fig. 7. Clustered column chart of the objectives values of suggested/current and proposed personnel assignment in the recruitment (REC) and reassignment problems (REA1, REA2 and REA3).

tions contain details of the experiments on reassignment and a discussion of the results.

2) Experiments on personnel reassignment: For each of the three reassignment scenarios, the management was asked to fill in a PCM to derive the priority of the objectives. Also, an expert in risk assessment configured the score functions for the global task-independent score (see (13)). As in the recruitment experiment, data related to each worker's human factors and strategy toward each risk were obtained from the worker's answers to the questionnaire. Pareto fronts were obtained by NSGA-II using the parameters in Table IV.

3) Discussion: All the companies saw global carefulness as one of the most important objectives (see Table III). The purpose of the experiments described in this section is to show that it is possible to significantly increase global carefulness just by reassigning some tasks to the most appropriate worker. The results demonstrate that the proposed framework can 
play a key role in SMEs, particularly in small manufacturing companies, which are very often small family realities with a low number of employees. In such companies, the capital available is often limited. Consequently, they tend to opt for low-cost solutions when attempting to improve safety and reduce the frequency with which workers are given tasks that they dislike.

a) REA1: REA1 relates to a factory with 8 employees. Global cost and global carefulness are the paramount objectives. The management therefore aims to improve workers' safety with a modest increase in costs. Global dislike is also not marginal, as shown by the weights in the second row in Table III.

The best solution is in Table V. Also, with the aid of Fig. 7, the performance of the proposed personnel assignment can be graphically compared to the current assignment strategy, by considering the second pair of clusters from the left. As can be seen, using the proposed framework, a significant increase $(78.35 \%)$ in the global carefulness at the expense of a $12.86 \%$ increase in the global cost was achieved. Note that the increase in cost is just temporary because it stems from training the reassigned workers.

Strategies with only high-level preventive actions increased from two to five. Also, there is no worker with a strategy with only low-level preventive actions for the assigned task, while the current assignment has three of these strategies, one of these with respect to a task with a hazardousness of 0.83 , where the worker's hands may be crushed. In addition, global dislike, i.e., the least important objective, is more than halved (from 7.44 to 2.45 ) with a percentage reduction of $67.06 \%$. The average dislike for the task is low and only one worker expresses a high dislike for the assigned task, while in the current assignment more than half of the workers express high and very high dislike values. Note that the improvements above are attainable by reassigning just three workers. Reassignments are shown in Fig. 8.

b) REA2: REA2 relates to a shoe factory with $10 \mathrm{em}-$ ployees. Similarly to REA1, REA2 is a scenario related to a company which considers global cost and global carefulness as being paramount. As can be seen from the third row in Table III, global cost has the greatest weight, suggesting that the company aims to increase global carefulness, but the economic resources are particularly limited. In REA2, global dislike also plays a very marginal role for the management. The results achieved are summarized in Table $\mathrm{V}$, and an immediate comparison with the current assignment is possible by looking at the third pair of clusters from the left in Fig. 7.

Thanks to the proposed reassignment, global carefulness achieves a $79.21 \%$ increase at the expense of just a temporary $5.24 \%$ increase in the global cost, which stems from the cost of training the reassigned workers, as can be surmised from (17). Strategies are now composed of only medium- and high-level preventive actions. Also, in the proposed assignment, there is no worker strategy with only low-level preventive actions, while the current assignment has five such strategies.

To achieve the aforementioned improvement in terms of global carefulness, global dislike is increased by 1.68 , in absolute terms. This is justifiable since the weight of dislike is 0.0372 , and six workers out of ten need to be reassigned (see Fig. 8). In any case, the increased global dislike is not dependent on workers having a dislike value for the assigned task lower than medium, in the current assignment.

c) REA3: REA3 relates to the largest of the factories involved, and employs 13 people. Two views of the scatter plot of the Pareto front of REA3 are shown, as representative of the personnel reassignment experiments, on the right hand side of Fig. 6, as (c) and (d).

Within REA3, global carefulness is the overriding objective, with no particular limitation on the increase in the global cost, which is very marginal. At the same time, the management is focused on employee satisfaction in terms of gratification gained from the task performed: in other words, the aim is also to achieve a low level of global dislike. The weights of the objectives for the REA3 scenario are in the last row in Table III. As shown, almost $97 \%$ of the importance is given to global carefulness and global dislike.

The current and proposed assignments are shown in Table V. The improvement can be easily evaluated by comparing
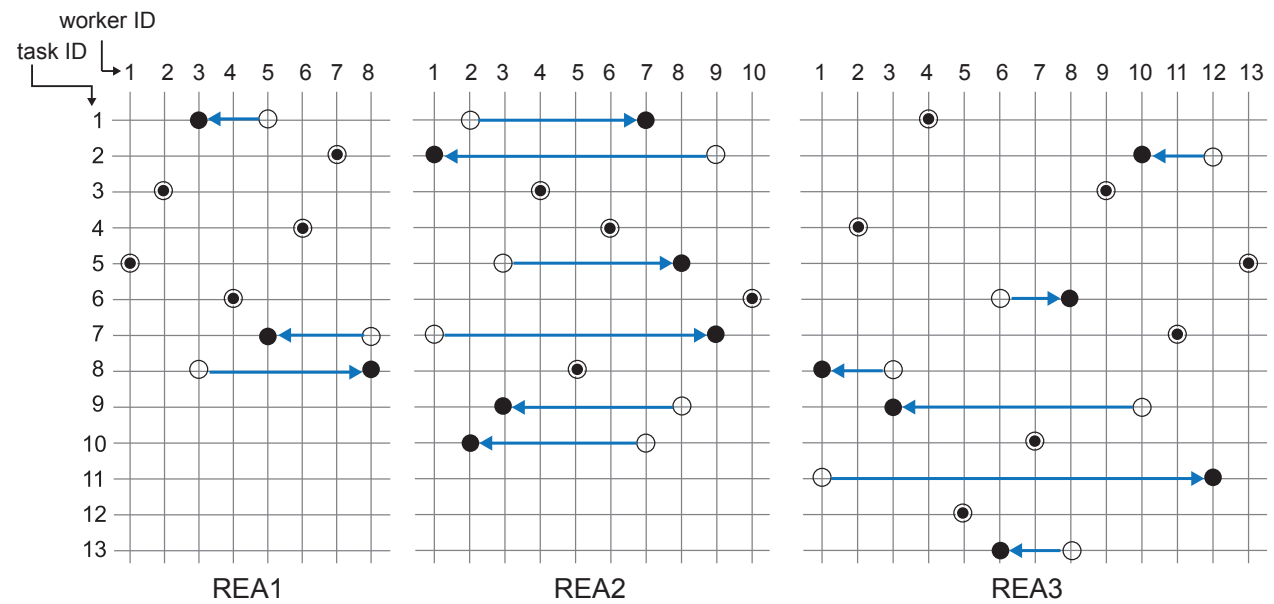

Fig. 8. (Re)assignment matrices for REA1, REA2 and REA3: empty and filled circles represent, respectively, current and proposed assignments; filled circles inside empty circles refer to not reassigned workers. Reassignments are represented as arrows directed from the current to the proposed assignment. 
the last two clusters in Fig. 7. In this scenario with no particular constraints on global cost, the proposed reassignment guarantees an $88.54 \%$ improvement in global carefulness. Strategies exclusively composed of low-level preventive actions are eliminated here. The current assignment has instead four tasks with a hazardousness greater than 0.7 performed by workers with strategies based on low-level preventive actions alone. The most dangerous of these tasks may lead to the worker's death as a consequence of falling from a loft where materials are stored. In the proposed reassignment, this task is performed by a worker with a strategy composed of only high-level preventive actions.

With reference to the global dislike, a $65.26 \%$ reduction is achieved: just 2 workers out of 13 have a medium dislike value for the assigned task; all the others have lower dislike values. In the current assignment, 6 workers have a dislike value of high and very high. Such significant improvements derive from six reassigned workers (see Fig. 8) which leads to a temporary $19.54 \%$ increase in the global cost for their training.

\section{COnClusion}

This paper has proposed a novel multi-objective formulation of the personnel assignment problem by exploiting the workers' sensitivity to risk. Because of people's subjectivity in perceiving hazardous situations, a novel measure, carefulness, was formalized. Carefulness stems from: (i) the accuracy of the precautions a worker takes when exposed to one or more risks; (ii) the values assumed by human factors related to the aspects influencing risk perception and propensity.

Considering a set of tasks and a set of workers, the proposed optimization model is focused on finding a Pareto-optimal personnel assignment that optimizes three objectives at the same time: maximizing the global carefulness, and minimizing the global cost and global dislike for the task assigned.

The problem was solved using a mixed EMO/MCDM resolution methodology. The NSGA-II algorithm was exploited to obtain an approximation of the Pareto front. A hybrid MCDM technique based on F-AHP and TOPSIS led to the selection of the Pareto-optimal solution representing the nearest compromise to the decision-maker's preferences.

Experiments were carried out thanks to 131 anonymous interviews provided by workers (or applicants) from four manufacturing SMEs, considering four real-world scenarios: one personnel recruitment with 10 workers, and three personnel reassignments with 8,10 and 13 workers, respectively.

The main novelty of the proposed framework consists in considering the workers' sensitivity to risk in the personnel assignment problem. Each task can therefore be assigned to the person with the safest interaction (i.e., the highest carefulness) with the task, thus making risks less likely and/or with fewer injuries. This is achieved by exploiting the workers' own capacity to prevent the risks of the tasks they perform.

The results showed that an outstanding increase in the global carefulness can be achieved just by reorganizing part of the worker-task associations, with no need for risk awareness training. Also, a high decrease in the overall workers' dislike for the task assigned, thus promoting a better work climate. These improvements are obtained at the expense of just a slight increase in the cost, which is paramount for SMEs.

Finally, thanks to the multi-objective formulation, our framework can be profitably exploited in work environments characterized by diverse levels of hazardousness, where safety may not represent the most crucial aspect to be optimized.

\section{REFERENCES}

[1] G. Parise, R. Nabours, and L. McClung, "Relevance of competence in risk reduction for electrical safety," IEEE Transactions on Industry Applications, vol. 44, no. 6, pp. 1892-1895, 2008.

[2] M. Keil, L. Wallace, D. Turk, G. Dixon-Randall, and U. Nulden, "An investigation of risk perception and risk propensity on the decision to continue a software development project," The Journal of Systems and Software, vol. 53, no. 2, pp. 145-157, 2000.

[3] M. Bouyer, S. Bagdassarian, S. Chaabanne, and E. Mullet, "Personality correlates of risk perception," Risk Analysis, vol. 21, no. 3, pp. 457-465, 2001.

[4] B. Chauvin, D. Hermand, and E. Mullet, "Risk perception and personality facets," Risk Analysis, vol. 27, no. 1, pp. 171-185, 2007.

[5] E. Peters and P. Slovic, "The role of affect and worldviews as orienting dispositions in the perception and acceptance of nuclear power," Journal of applied social psychology, vol. 26, no. 16, pp. 1427-1453, 1996.

[6] L. Sjöberg, "Factors in risk perception," Risk Analysis, vol. 20, no. 1, 2000.

[7] P. Slovic, "Perception of risk," Science, vol. 236, no. 4799, pp. 280-285, 1987.

[8] D. Sharma, B. Alford, S. Bhuian, and L. Pelton, "A higher-order model of risk propensity," Journal of Business Research, vol. 62, pp. 741-744, 2009.

[9] S. Sitkin and L. Weingart, "Determinants of risky decision-making behavior: a test of the mediating role of risk perception and propensity," Academy of management, vol. 38, no. 6, pp. 1573-1592, 1995.

[10] A. Sueiro, I. Sánchez-Iglesias, and A. M. de Tella, "Evaluating risk propensity using an objective instrument," The Spanish Journal of Psychology, vol. 14, no. 1, pp. 392-410, 2011.

[11] M. S. Horswill and F. P. McKenna, "The effect of perceived control on risk taking," Journal of Applied Social Psychology, vol. 29, no. 2, pp. 377-391, 1999.

[12] C. T. F. Klein and M. Helweg-Larsen, "Perceived control and the optimistic bias: a meta-analytic review," Psychology and health, vol. 17, no. 4, pp. 437-446, 2002.

[13] J. Costa-Font, E. Mossialos, and C. Rudisill, "Optimism and the perception of new risks," Journal of risk research, vol. 12, no. 1, pp. 27-41, 2009.

[14] B. Lazzerini and F. Pistolesi, "Profiling risk sensibility through association rules," Expert Systems with Applications, vol. 40, no. 5, pp. 1484 1490, 2013.

[15] K.-Y. Teng, S. Thekdi, and J. Lambert, "Risk and safety program performance evaluation and business process modeling," IEEE Transactions on Systems, Man, and Cybernetics - Part A: Systems and Humans, vol. 42, no. 6, pp. 1504-1513, 2012.

[16] B. Lazzerini and F. Pistolesi, "Classifying workers into risk sensibility profiles: a neural network approach," in 2014 European Modelling Symposium, Pisa, IT, 2014, pp. 33-38.

[17] M. Cococcioni, B. Lazzerini, and F. Pistolesi, "A semi-supervised learning-aided evolutionary approach to occupational safety improvement," in IEEE Congress on Evolutionary Computation, Vancouver, CA, 2016, pp. 3695-3701.

[18] S. Bandini, S. Manzoni, and F. Sartori, "Case-based reasoning to support work and learning in small and medium enterprises," in 21st IEEE International Conference on Tools with Artificial Intelligence, Newark, NJ, USA, 2009, pp. 253-260.

[19] T. Saaty, "How to make a decision: The analytic hierarchy process," European Journal of Operational Research, no. 48, pp. 9-26, 1990.

[20] T. L. Saaty, "Decision-making with the AHP: Why is the principal eigenvector necessary," European Journal of Operational Research, vol. 145, no. 1, pp. 85-91, 2003.

[21] J. E. Smith and D. von Winterfeldt, "Anniversary article: Decision analysis in management science," Management Science, vol. 50, no. 5 , pp. 561-574, 2004.

[22] L. A. Zadeh, "Fuzzy sets," Information and Control, vol. 8, no. 3, pp. 338-353, 1965 . 
[23] K. Y. Ching-Lai Hwang, Multiple attribute decision making. SpringerVerlag, 1981.

[24] K. Yoon, "A reconciliation among discrete compromise situations," Journal of Operational Research Society, vol. 38, pp. 277-286, 1987.

[25] F. Liu and W.-G. Zhang, "TOPSIS-based consensus model for group decision-making with incomplete interval fuzzy preference relations," IEEE Transactions on Cybernetics, vol. 44, no. 8, pp. 1283-1294, 2014.

[26] B. Lazzerini and F. Pistolesi, "Neural network-based objectives prioritization for multi-objective economic dispatch in microgrids," in 2014 IEEE/SICE International Symposium on System Integration, Tokyo, JP, 2014, pp. 665-671. [Online]. Available: http://dx.doi.org/10. 13140/2.1.5142.0960

[27] J. H. Holland, Adaptation in Natural and Artificial Systems: An Introductory Analysis with Applications to Biology, Control and Artificial Intelligence. Cambridge, MA, USA: MIT Press, 1992.

[28] D. E. Goldberg and R. Lingle, Jr., "Alleles loci and the traveling salesman problem," in Proceedings of the 1st International Conference on Genetic Algorithms, Hillsdale, NJ, USA, 1985, pp. 154-159.

[29] K. Deb, A. Pratap, S. Agarwal, and T. Meyarivan, "A fast and elitist multiobjective genetic algorithm: NSGA-II," IEEE Transactions on Evolutionary Computation, vol. 6, no. 2, pp. 182-197, 2002.

[30] D. J. Jakobczak, Analyzing Risk through Probabilistic Modeling in Operations Research. IGI Global, 2016.

[31] K. P. Sachin and R. Kant, "A fuzzy AHP-TOPSIS framework for ranking the solutions of knowledge management adoption in supply chain to overcome its barriers," Expert Systems with Applications, vol. 41, no. 2, pp. 679-693, 2014.

[32] G. Buyukozkan and G. Cifci, "A combined fuzzy AHP and fuzzy TOPSIS based strategic analysis of electronic service quality in healthcare industry," Expert Systems with Applications, vol. 39, no. 3, pp. 23412354, 2012.

[33] R. Marler and J. Arora, "Survey of multi-objective optimization methods for engineering," Structural and Multidisciplinary Optimization, vol. 26, no. 6, pp. 369-395, 2004

[34] M. Velasquez and P. T. Hester, "An analysis of multi-criteria decision making methods," International Journal of Operations Research, vol. 10, no. 2, pp. 56-66, 2013.

[35] W. Zung, "A rating instrument for anxiety disorders," Psychosomatics, vol. 12 , no. 6 , pp. $371-379,1971$.

[36] T. Meyer, "Development and validation of the Penn State Worry Questionnaire," Behaviour Research and Therapy, vol. 28, no. 6, pp. $487-495,1990$.

[37] M. Rosenberg, Society and the adolescent self-image. Wesleyan University Press, 1989.

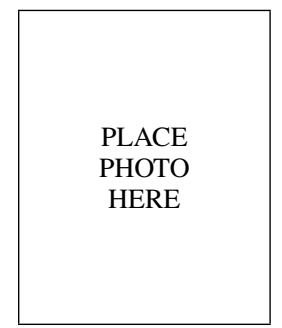

Beatrice Lazzerini (M'98) is a Full Professor of computer engineering with the Department of Information Engineering, University of Pisa, Pisa, Italy. She has coauthored seven books and has contributed to more than 210 papers in international journals and conferences. She is coeditor of two books. She was involved and had roles of responsibility in several national and international research projects and scientific events. Her research interests include computational intelligence, with a particular emphasis on fuzzy systems, neural networks, and evolutionary computation, and their applications to pattern classification, risk analysis and management, diagnosis, forecasting, and multicriteria decision making.

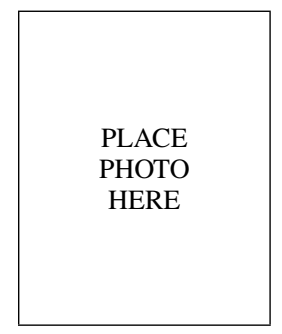

Francesco Pistolesi received the Graduate degree (summa cum laude) in computer engineering (curriculum enterprise systems) and Ph.D. degree in information engineering (curriculum computer system architectures) from the University of Pisa, Pisa, Italy. $\mathrm{He}$ is currently a Postdoctoral Researcher with the Department of Information Engineering, University of Pisa. His research interests include computational intelligence and data mining, with applications to decision support and multiobjective optimization. His current research activity is mainly focused on innovative solutions for smart industries, from industrial processes to occupational health and safety. 